
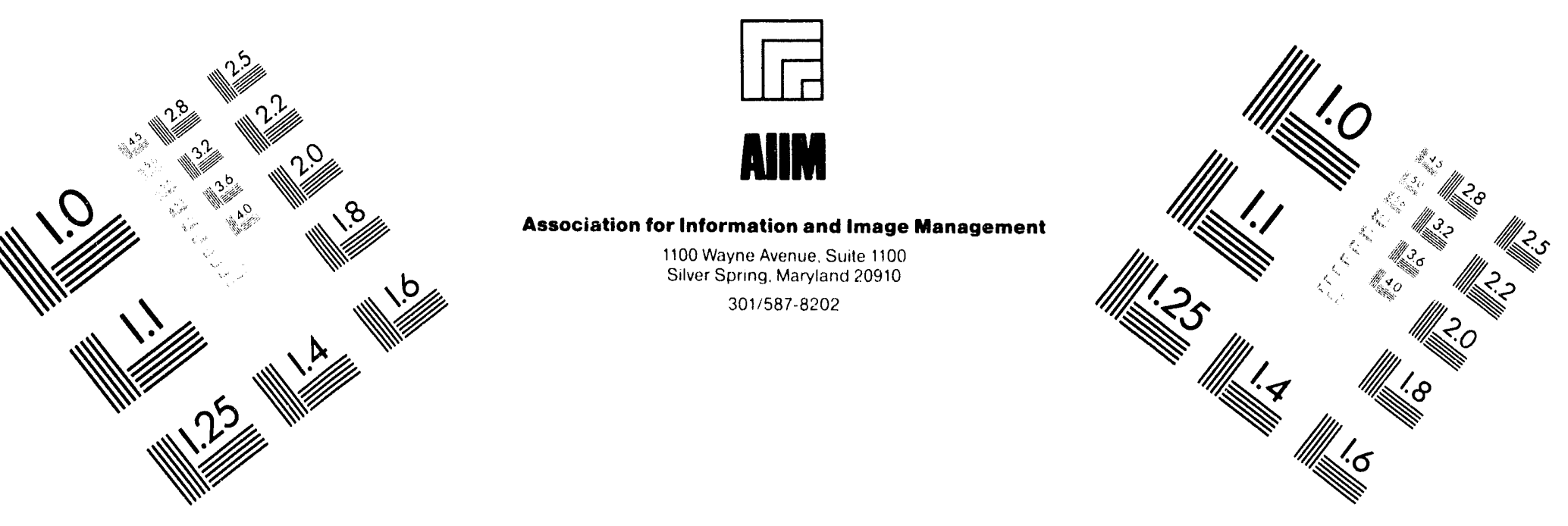

\title{
Centimeter
}

$\mid \begin{aligned} & 1 \\ & \mid\end{aligned}$

Inches
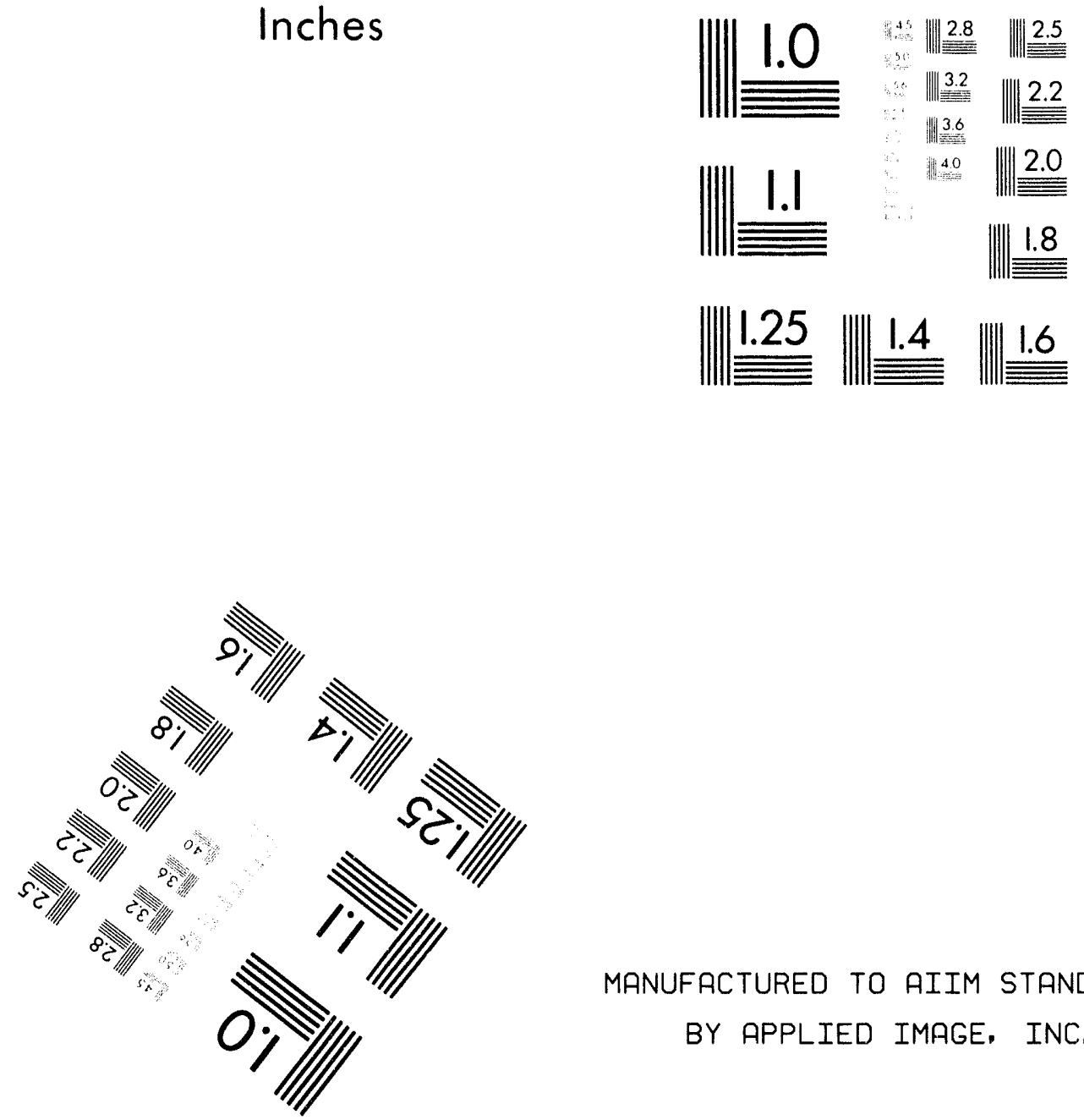

MANUFACTURED TO AIIM STANDARDS

BY APPLIED IMAGE. INC.

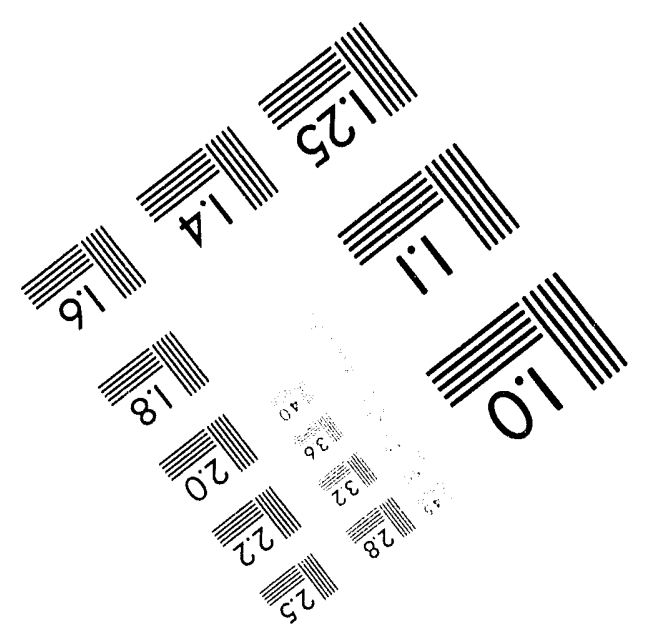




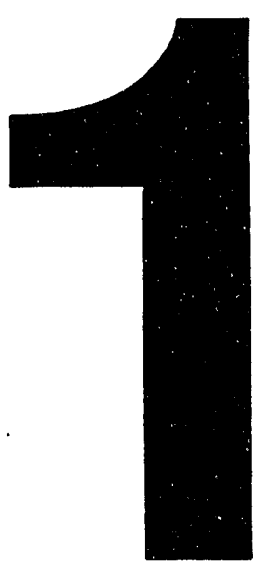




\section{MIDWEST SUPERCONDUCTIVITY CONSORTIUM 1993 Progress Report}

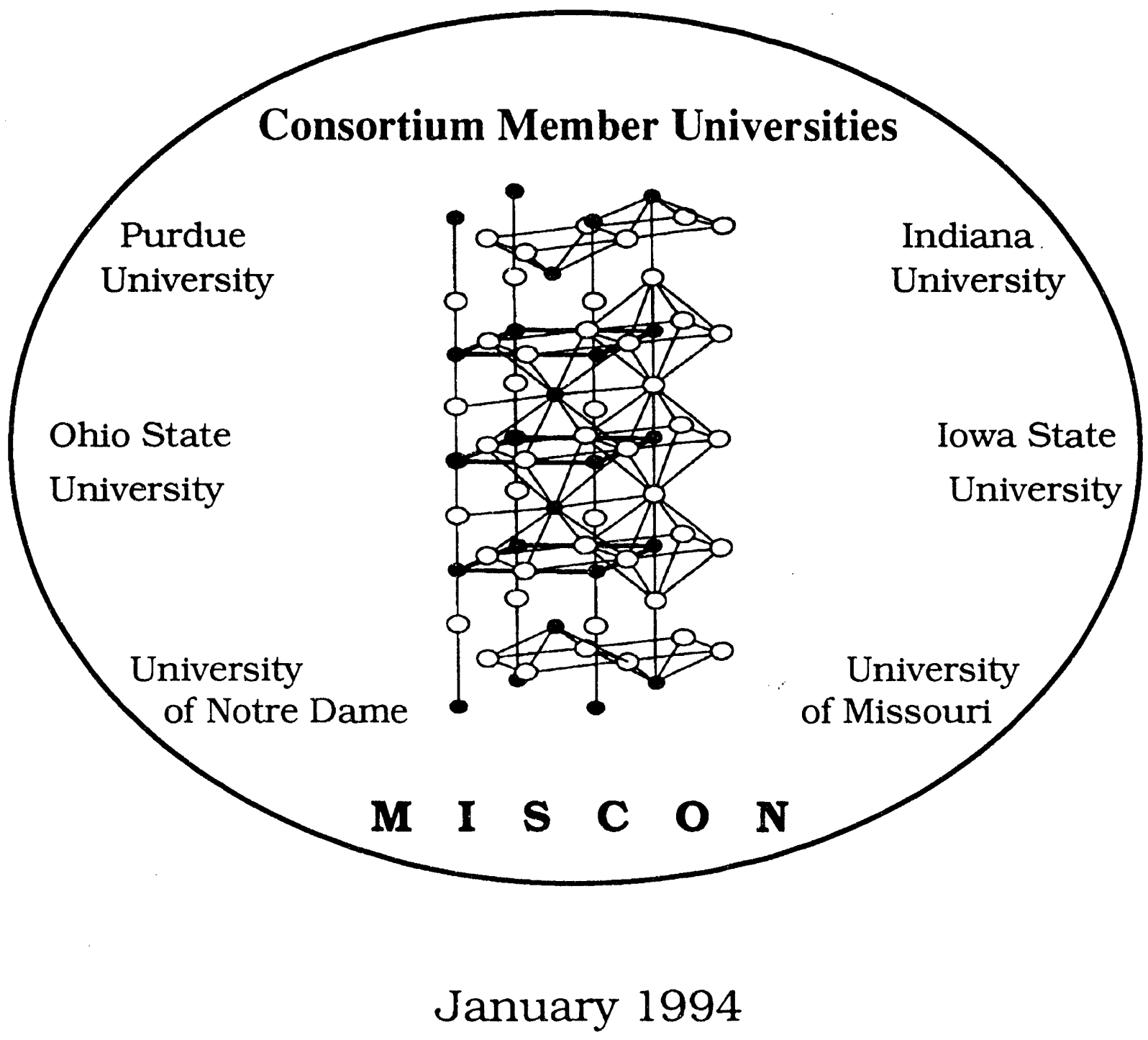

Submitted to the:

U. S. Department of Energy Grant No. DE-FGO2-90ER45427 DOE Report No. DOE/ER/45427-4

Arden L. Bement, Jr., Director Purdue University West Lafayette, IN 47907 DISTRIBUTION OF THIS DOCUMENT IS UNLIMITED 


\title{
Midwest Superconductivity Consortium
}

\author{
SPONSOR: \\ Division of Materials Sciences \\ Office of Basic Energy Sciences \\ United States Department of Energy
}

PARTICIPATING UNIVERSITIES:

Indiana University

Iowa State University

University of Missouri - Columbia

University of Notre Dame

Ohio State University

Purdue University

PARTICIPATING AFFILIATES:

Advanced Technology Materials, Inc.

AT\&T Bell Laboratories

Bellcore

IBM Thomas J. Watson Research Center

Intermagnetics General Corporation

Lake Shore Cryotronics, Inc.

National Institute of Standards and Technology

Quantum Magnetics/Quantum Design

Ribbon Technology Corporation

TRW Superconductivity Research

Weizmann Institute of Science 
Page

1. Summary................................................................................................... 1

2. Program Overview................................................................................... 4

3. Research Program Thrusts .................................................................. 7

4. Research Program ................................................................................ 8

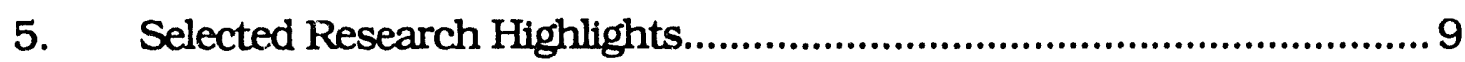

6. Talks and Presentations .....................................................................37

7. Publications

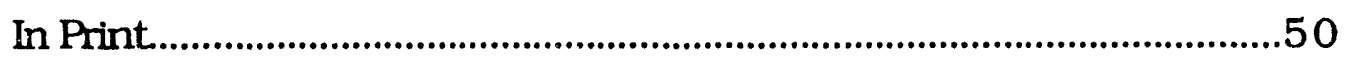

Accepted for Publication ............................................................................57

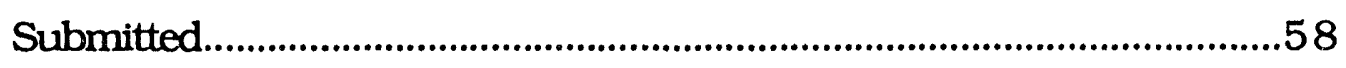

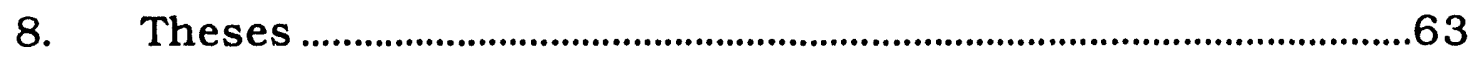

Appendix I. - Research Program Listing...............................................64

Appendix II. - 1992 Cooperative Interactions.......................................68

Appendix III. - Organization .............................................................................83 


\section{SUMMARY}

The mission of the Midwest Superconductivity Consortium, MISCON, is to advance the science and understanding of high $\mathrm{T}_{c}$ superconductivity.

During the past year, twenty-seven (27) projects produced over one hundred thirty-five (135) talks and one hundred twenty-six (126) publications. Four (4) Master's Degrees and five (5) Doctor's of Philosophy Degrees were granted to students working on MISCON projects.

Group activities and interactions involved two (2) MISCON group meetings (held in July and January); the first MISCON Workshop held in July; sixteen (16) external speakers; sixty-four (64) collaborations (with universities, industry, Federal laboratories, and foreign research centers); and twenty-three (23) exchanges of samples and/or measurements.

Research achievements this past year focused on understanding the effects of processing phenomena on structure-property interrelationships and the fundamental nature of transport properties in high-temperature superconductors.

In the synthesis and processing area: new low-temperature routes for processing bulk and thin film materials were developed; understandings of crystallization, grain growth and texture development in solidified melts and deposited thin films were broadened; important effects of substitutions, second phases, texture alignments, modulated microstructures and composite and microlaminate structures on superconducting properties were characterized; and, processing routes for improving the density and texture of tubular shielding structures were explored.

In the area of transport phenomena: theories for universal conductivity at superconductor-insulator transitions, flux-lattice melting transitions, the electrodynamics and thermodynamics of vortex motion. electromagnetic wave dissipation, and Josephson current densities through insulating barriers were advanced; important experimental techniques for measuring magneto-transmission and reflections of $\mathrm{mm}$ microwaves, vortex correlation fluctuations, magnetic penetration depths, the softening of in-plane phonon modes, flux pinning by complex defects 
and interfaces, weak-link behaviors, and reproducible sub-energy-gap structures were studied.

The following are examples of notable achievements by MISCON investigators during the year:

- YBCO processed by the solid-liquid melt growth (SLMG) process form textured 123 phase with fine (ca 100nm) 211-phase precipitates. These samples exhibit extremely good low temperature flux pinning properties. Argonne National Laboratory has filed a patent application for this process. (P. McGinn, University of Notre Dame, E. Kvam, Purdue University, and D. Shi, Argonne National Laboratory).

- A formaiism which for the first time makes fully microscopic calculations of the mixed state has been developed and is being applied to calculations of the rilicrowave conductivity. (A. H. MacDonald and S. M. Girvin. Indiana University).

- The flux-lattice melting temperature of single-crystal YBCO has been calculated without any adjustable parameters, in excellent agreement with experimental results. (D. G. Stroud, Ohio State University).

- Superconducting junction arrays, consisting of $300 \times 300$ proximitycoupled, weak-link junctions fabricated of $\mathrm{Ag} / \mathrm{YBa}_{2} \mathrm{Cu}_{3} \mathrm{O}_{7}$ on $\mathrm{SrTiO}_{3}$ substrates, have been fabricated and characterized. These junctions exhibit a Kosterlitz-Thouless-type transition in the superconducting state. (J. C. Garland, Ohio State University).

- Some key superconducting characteristics for BSCCO have been extracted from the theory of thermal fluctuations of vortices in layered superconductors. These properties include the temperature dependencies of the penetration depth, $\lambda_{\mathrm{ab}}$, the upper critical field, $\mathrm{H}_{\mathrm{c} 2}$. and the Ginzberg-Landau parameter, א. (J. R. Clem and V. G. Kogan, Iowa State University).

- The first direct measurement of a vortex lattice in a superconducting niobium film was demonstrated by measuring NMR transitions in an 
atomic beam directed across the surface of the film. (G. Lafyatis and T. Lemberger, Ohio State University).

- The thermal decomposition of 124 leads to the eventual formation of $211, \mathrm{Cu}_{2} \mathrm{BaO}_{2}$, and other phases under a three-step process. In contrast to earlier work, the decomposition of 124 , per se, takes place in one step with a characteristic activation energy. (D. V. Baxter, K. G. Caulton, and M. H. Chisholm, Indiana University).

- The first direct determination of the magnetic penetration depth was made for an oxygen-depleted YBCO film. The results reveal that the penetration depth increases with oxygen depletion, but that its temperature dependence is unchanged, consistent with $d$-wave superconductivity. (T. R. Iemberger, Ohio State University).

- A three-tunnel capacitor model for single-electron tunneling was successfully applied to explain the highly non-linear I-V characteristics obtained with T1Ba2 (Ca0.8Y0.2) $\mathrm{Cu}_{2} \mathrm{O}_{7}$ films. (M. W. McElfresh and R. G. Reifenberger, Purdue University).

- A new, direct method has been developed for analyzing magnetic relaxation data. With this method it is now possible to determine the dependence of the pinning potential, $U(\mathrm{~m})$, of the magnetic induction, $B$, as well as to see the effects of oxidation-induced pinning centers on U(m). (W. B. Yelon and J. W. Farmer, University of Missouri).

- Two vortex phase transitions have been observed for Tl-2212. The lcwer temperature transition corresponds to a vortex-glass transition while the higher temperature transition corresponds to a pancake-vortex coupling transition. (M. W. McElfresh and R. G. Reifenberger, Purdue University).

- Measurements of 2-dimensional $89 \mathrm{Y}$ NMR in $\mathrm{YBa}_{2} \mathrm{Cu}_{3} \mathrm{O}_{7}$ to probe vortex dynamics reveal that in fields of several Tesla, vortices do not make spatial jumps of distances comparable to the lattice spacing. (C. Pennington, Ohio State University). 
- The first Raman scattering studies of the phonon modes of $\mathrm{YBa}_{2} \mathrm{Cu}_{4} \mathrm{O}_{8}$ (Y124) under pressure have been made. The mode Grüeisen parameters of the phonons present in both Y124 and Y123 scale with their bulk moduli. However, the chain modes that are Raman allowed only in Y124 are anomalously different. (M. Chandrasekhar, University of Missouri).

- Improvements have been made to a leadless, nondestructive microwave technique for characterizing high-temperature superconductors in the presence of a sustained dc magnetic field (0-7 Tesla). It is now possible to infer with reasonable agreement microwave surface impedance directly from the data. (W. J. Tomasch and J. K. Furdyna, University of Notre Dame).

- For YBCO with 211 additions, the enhancement of the critical current through flux pinning has been found to depend on the size of the 211 particles. However, the pinning seems to be induced more by extended defects (dislocations and $\mathrm{CuO}$ layer stacking faults) associated with the particles rather than by the particles themselves. (E. Kvam, Purdue University).

\section{PROGRAM OVERVIEW}

The Midwest Superconductivity (jonsortium, MISCON, in the fourth year of operations further strengthened its mission to advance the science and understanding of high Tc superconductivity. The goals of the organization and the individual projects continue to reflect the current needs for new knowledge in the field and the unique capabilities of the institutions involved. Group rfforts and cooperative laboratory interactions to achieve the greatest possi'sle synergy under the Consortium continue to be emphasized. Industrial affiliations coupled with technology transfer initiatives were expanded. Activities of the participants during the past year achieved an interactive and high level of performance. The number of notable achievements in the field contributed by Consortium investigators increased.

The programmatic research continues to focus upon key materialsrelated problems in two areas. The first area has a focus upon "Synthesis 
and Processing" while the second is centered around "Limiting Features in Transport Properties of High $T_{c}$ Materials". The two areas involve twentyseven (27) research projects and thirty-eight (38) principal investigators. The research projects involve seven (7) undergraduate students, fifty-seven (57) graduate students and twelve (12) postdoctoral associates. In the past year, these participants have published sixty-three (63) papers. Nine (9) more papers are in press and an additional fifty-four (54) have been submitted. Further, the participants have given over one hundred thirtyfive (135) talks or conference presentations. During this past year, four (4) Master's Degrees and five (5) Doctor's of Philosophy degree were granted to students working on MISCON projects.

Group activities and interactions with others were again a major activity under the Consortium. MISCON group meetings were held in January and July. A two day schedule for each meeting provided ample opportunity for the desired interactions to take place. Based upon these meetings the Program Committee reexamined the goals and programmatic research and made desirable program changes.

In addition to the group meetings, a workshop entitled "Type-II Superconductors: Basic Properties, Measurements, and Selected Advanced Topics", was held on July 8-9, 1993, to provide a tutorial on leading theories and experimental developments in transport behavior. Outstanding speakers from both within and outside MISCON participated. Underwriting support was provided by Quantum Design and Lake Shore Cryotronics, Inc., two of MISCON's Affiliates.

Coupled with the group meetings and workshop, the participants were very active in cooperative interactions with MISCON Affiliates and other organizations. Among these activities were sixteen (16) external speakers, sixty-four (64) collaborations, twenty-three (23) exchanges of samples and/or measurements, and one (1) gift of equipment from industry. The number of actively participating industrial Affiliates increased by 3 (from 8 to 11) with the addition of Bellcore, Lake Shore Cryotronics, Inc., and The Weizmann Institute of Science. Additional affiliations are pending.

Group activities are fostered by three programs: first, a seminar exchange promotes interaction within and between the groups; second, a programmatic exchange facilitates the use of special capabilities by all 
participants; finally, a special capital equipment competition addresses group needs as the primary criterion. Cost sharing realized over $\$ 234 \mathrm{~K}$ worth of equipment, that was selected to provide new capabilities to the consortium, with less than $\$ 118 \mathrm{~K}$ of MISCON funds invested. One (1) equipment gift was received from industry.

Industrial affiliations and technology transfer activities were expanded during the year. Nine (9) written agreements and two (2) equipment access agreements were formalized this year with eleven (11) different industry and national laboratory groups. Seven (7) additional formal agreements are pending. Such interactions make all paiticipants more aware of industry's needs for new knowledge and the current state of the technology.

\section{PEER REVIEW}

In accordance with MISCON procedures all fourth-year proposals and new proposals are reviewed by an independent external peer review panel. The 1993 peer review was conducted by nine outstanding experts in the field of high- $T_{C}$ superconductivity. Project renewal decisions were then based on the results of this peer review and the 1992 DOE/OPA review. Two projects were directed to be phased out based on low scores in both reviews, and two projects were significantly redirected to refocus the work on those elements given higher marks by the reviewers. The mean score for the remaining projects was near the midpoint between "very good" and "excellent", representing a significant improvement in the overall quality of the program.

In addition to the fourth-year reviews, six new project proposals were peer reviewed. Of these, four scored sufficiently high to justify funding for 1993/94. These were funded at a reduced level for the first year for a total of $\$ 212,628$.

Finally, two projects were voluntarily withdrawn; for one the goals had been completed. These changes in research projects are indicated in Appendix I. 
The process of peer review will continue to be the principal means within MISCON for continuous improvement of project quality, productivity and synergy and for dynamic renewal of project compositions within the two main program thrusts.

\section{RESEARCH PROGRAM THRUSTS}

MISSION: The basic mission of the Consortium is to advance the science and understanding of high- $T_{c}$ superconductivity and to promote the development of new materials and improved processing technology for the commercialization of superconductivity.

GOALS: The goals of the Consortium are:

(a) To develop, utilizing effective group efforts, the scientific and technological foundation for the industrial applications and commercialization of high-temperature superconductivity;

(b) To facilitate the transfer of technology from the Consortium research laboratories into the industries of the Midwest; and

(c) To ensure that there is a continuing supply of engineers and scientists trained in this important field.

PROGRAMMATIC RESEARCH: To achieve the research goals within the mission of the Consortium, two general programmatic areas of research have been defined. These two areas were selected from many possible ones to focus upon key materials-related problems where unique capabilities exist within the Consortium. In addition to cooperative research programs in these areas, the Consortium encourages proposals for studies of new materials which can be expected to exhibit useful hightemperature superconductivity. 
Area 1: New and Improved High $T_{c}$ Superconducting Materials: Phase Relations, Reactions, Synthesis and Processing

The development of high-temperature superconductors is contingent upon a thorough understanding of their material properties and of the processing variables that control these properties. Such properties include both thermodynamic and kinetic information about superconducting and normal-state phases. Processing variables encompass those necessary for optimizing synthesis and processing procedures for materials properties control. The Consortium supports investigation of these topics, as well as studies pertaining to the fabrication and characterization of high-temperature superconducting composites, multilayers, modulated structures, thin films, and other novel structures of potential usefulness.

Area 2: High Tc Superconductor Transport Properties:

\section{Controlling/Limiting Factors}

Most proposed applications of high-temperature superconductors involve their ability to carry bulk supercurrents (e.g., wires, ribbons), to screen magnetic fields (e.g., enclosures, cavities), or to be components of electronic devices (e.g., sensors, magnetometers). All of these applications are contingent upon an understanding and control of transport properties. The Consortium supports research in this general area, including studies of weak links (both artificially produced, and naturally occurring, as by defects, grain boundaries and textures); dynamics (flux lattice pinning, melting, and related phenomena); and interfacial transport (such as that between grains, films, contacts, substrates, and coatings).

\section{RESEARCH PROGRAM}

Titles of the projects within the two principal research program areas are given in Appendix I, along with industrial affiliations of the principle investigators for each project. A major emphasis during the past year was to increase the number of affiliations with other organizations. Eight (8) out of twenty-seven (27) projects now involve formalized working agreements. Those affiliations covered by formalized agreements are also indicated in Appendix I. 


\section{SELECTED RESEARCH HIGHIGHTS}

The following are selected research accomplishments by MISCON participants in the past year. These achievements provide an insight into some of the new and exciting areas of research in progress by MISCON participants. Formal affiliations are noted by an asterisk.

Area 1: New and Improved High $T_{c}$ Superconducting Materials: Phase Relations, Reactions, Synthesis and Processing

Low Temperature Routes to Processing of High-Temperature Superconductors

David V. Baxter, Kenneth G. Caulton, and Malcolm H. Chisholm. Indiana University Advanced Technology Materials, Inc.*

The objectives of this research project are:

1. To elucidate the mechanisms through which molecular precursors are transformed into superconducting materials during the CVD process;

2. To study the phase transformations in the YBCO system; and

3. To use this knowledge to improve the design of new generations of precursors.

The gas handling system for our Remote Plasma Enhanced Chemical Vapor Deposition (RPECVD) reactor has been completed. Preliminary studies of the process pumping capability, plasma generator, and mass spectrometry system (including differential pumping) reveal that these various subsystems all perform as expected.

Thermal decomposition studies of 124 show a three-step process leading to the eventual formation of $211, \mathrm{Cu}_{2} \mathrm{BaO}_{2}$ and other phases. In contrast to earlier work we find that the decomposition of the 124 phase 
takes place in one step, and that the associated process is consistent with an activation barrier of $2.7 \mathrm{eV} /$ particle.

We have completed our characterization of compounds of barium and copper coordinated by a new class of ligand and the addition of a donor base. This work has been submitted for publication. Molecular modeling graphics, based on our structure determinations, has given us the means to select and synthesize a new improved copper-containing precursor. The compound $\mathrm{Cu}(\mathrm{B})_{2}$ is promising with regard to its volatility without the necessity of added donor bases, and thus this theme will be emphasized.

\section{Study of Compositional Modulation in High-Tc Superconductor Structures and Its Influence on their Superconducting Properties. \\ K. M. Choudhary and S. T. Ruggiero University of Notre Dame}

The objectives of this project are to study the fabrication and characterization of HTSC composites, multilayers, modulated structures, thin films, and other useful structures employing the following specific approaches:

1. Development of newly discovered ErBaCuO compounds;

2. Productions of HTSC/AMnO3 multilayers; and

3. Introduction of controlled defects to pin flux and thereby increase $J_{c}$.

Results during the year include:

- Orthorhombic-DyMnO3 thin films were synthesized by molecular beam epitaxy. This is another classic example of metastable phase synthesis by MBE. In a bulk form, the orthorhombic phase of DyMnO3 can only be prepared under high pressure.

- Epitaxial orthorhombic-DyMnO3(110)/ $\mathrm{Er}_{1} \mathrm{Ba}_{2} \mathrm{Cu}_{3} \mathrm{O}_{7-\delta} \delta(001)$ and orthorhombic-DyMnO3(110)/Er5Ba7 $\mathrm{Cu}_{12} \mathrm{O}_{\mathrm{y}}(001)$ thin film heterostructures on $\mathrm{LaAlO}_{3}(100)$ substrates were successfully grown. 
Therefore, the feasibility of fabricating $\mathrm{DyMnO}_{3}(110) / \mathrm{Er}_{1} \mathrm{Ba}_{2} \mathrm{Cu}_{3} \mathrm{O}_{7}$ $\delta(001)$ superlattices has been demonstrated.

- In comparison with the $\mathrm{Er}_{1} \mathrm{Ba}_{2} \mathrm{Cu}_{3} \mathrm{O}_{7-\delta}(001)$ thin films, the surface smoothness of the [001] oriented new $\mathrm{Er}_{5} \mathrm{Ba}_{7} \mathrm{Cu}_{12} \mathrm{O}_{\mathrm{y}}$ hightemperature superconductor thin films was found to be better. Furthermore, the transport properties of the $\mathrm{Er}_{5} \mathrm{Ba}_{7} \mathrm{Cu}_{12} \mathrm{O}_{\mathrm{y}}(001)$ and $\mathrm{Er}_{1} \mathrm{Ba}_{2} \mathrm{Cu}_{3} \mathrm{O}_{7-\delta}(001)$ thin films were found to be similar. These results suggest that the $\mathrm{Er}_{5} \mathrm{Ba}_{7} \mathrm{Cu}_{12} \mathrm{O}_{\mathrm{y}}(001)$ thin films may have potential for applications in superconductor electronics.

\section{Microstructural Dependence of Critical Current Density in Melt Textured $\mathrm{YBa}_{2} \mathrm{Cu}_{3} \mathrm{O}_{6+x}$ Superconductors \\ P. J. McGinn \\ University of Notre Dame \\ Argonne National Laboratory*}

The goal for this project is to investigate the microstructural dependence of $J_{C}$ in zone-melt-textured YBCO so as to optimize its microstructure and improve its properties and processability.

Studies were performed on $\mathrm{Y}_{2} \mathrm{BaCuO}_{5}(211)$ and liquid $\left(\mathrm{L}=3 \mathrm{BaCuO}_{2}: 2 \mathrm{CuO}\right)$ to study the effect of additions of $\mathrm{BaSnO}_{3}$ and $\mathrm{PtO}_{2}$ on the coarsening behavior of 211 particles in the liquid above the peritectic temperature of $\mathrm{YBa}_{2} \mathrm{Cu}_{3} \mathrm{O}_{6+\mathrm{x}}(123)$ and the magnetic properties after melt texturing.

Some key results are:

- The solid-liquid melt growth (SLMG) process for producing textured 123 with fine $(-100 \mathrm{~nm}) 211$ has been developed. Samples processed by this route exhibit extremely good low temperature flux pinning properties. A patent application has been filed by Argonne National Laboratory. 
- Studies have shown that although additions of $\mathrm{BaSnO}_{3}$ and $\mathrm{PtO}_{2}$ both reduce the coarsening of $211, \mathrm{PtO}_{2}$ does so much more effectively. The $\mathrm{PtO}_{2}$ additions refine 211 by altering the 211 /liquid interfacial energy and/or $\mathrm{Y}$ diffusivity, and not by acting as heterogeneous nucleation sites as reported in some studies in the literature. $\mathrm{BaSnO}_{3}$ additions are found to not be inert in the melt as reported by others.

- It has been found that $\mathrm{PtO}_{2}$ additions lead to an increase in the defect density in textured 123, and it is this change in the defect level which appears to be the source of the enhanced pinning and higher $\mathrm{J}_{\mathrm{c}}$ observed with $\mathrm{PtO}_{2}$ (or Pt) additions.

\section{Growth of Single Crystals and/or Textured Specimens of High-Tc Superconductors using Oxygen Seeding Patrick K. Gallagher The Ohio State University}

The objective of this research project is to demonstrate the efficacy of oxygen seeding for the growth of single crystal or highly textured $\mathrm{YBa}_{2} \mathrm{Cu}_{3} \mathrm{O} 7$ (123).

Several techniques for oxygen seeding have been tried. Although the success has been limited, it is encouraging and indicates that preferred nucleation and growth based on seeding with oxygen is a viable technique for YBCO (123). Preliminary studies also indicate that it can also be achieved in the BSCCO system. 


\section{Processing of Textured Superconducting Oxide/Noble Metal Microlaminates \\ Ken H. Sandhage \\ The Ohio State University \\ P. J. McGinn \\ University of Notre Dame \\ Intermagnetics General Corporation* \\ Ribbon Technology Corporation*}

The objective of this research program is to produce fracture-tough, high $J_{C}$ superconducting composites consisting of thin layers of superconducting oxide alternating with thin layers of noble metal.

Research accomplishments include:

- Attempts to melt texture $\mathrm{YBa}_{2} \mathrm{Cu}_{3} \mathrm{O}_{7-\mathrm{y}}$ on $\mathrm{Ag}$ substrates (or in a silver crucible) in a reduced oxygen partial pressure at temperatures below the Ag melting point resulted in consumption of the Ag by the peritectic liquid.

- A $90 \mathrm{wt} \% \mathrm{Ag} / 10 \mathrm{wt} \% \mathrm{Pd}$ alloy is only minimally attacked by the peritectic liquid. Successful melt texturing of $\mathrm{YBa}_{2} \mathrm{Cu}_{3} \mathrm{O}_{7-\mathrm{y}}$ at temperatures below $955^{\circ} \mathrm{C}$ in an alloy crucible has been performed. This suggests that texturing of microlaminates is possible.

- While chemically-homogeneous metallic precursor tapes have been produced by spinning molten $\mathrm{Y}-\mathrm{Ba}-\mathrm{Cu}-\mathrm{Ag}$ alloys, it is difficult to obtain melt-spun ribbons with flat surfaces. However, dense, chemically-uniform precursor tapes have been produced by hot rolling of mechanically-alloyed $\mathrm{YBa}_{2} \mathrm{Cu}_{3} \mathrm{Ag}_{46} \mathrm{Pd}_{4.7}$ powder inside a silver tube.

- 200 micron thick YBa2Cu3Ag46Pd4.7 tapes can be completely oxidized within $\approx 7$ hours at $400^{\circ} \mathrm{C}$ in oxygen, suggesting that the synthesis of microlaminates of $\mathrm{YBa}_{2} \mathrm{Cu}_{3} \mathrm{O}_{7}-\mathrm{y} / \mathrm{Ag}-\mathrm{Pd}$ within reasonable times is possible. 


\section{Anisotropic Grain Growth and Isothermal Grain Alignment of Bulk, Superconducting Oxides \\ Ken $\boldsymbol{H}$. Sandhage \\ The Ohio State University American Superconductor Corp.*}

The goals of this research program are:

1. To understand the influence of various processing parameters (temperature, composition, oxygen partial pressure) on the grain growth of superconducting axides; and

2. To tailor the growth of superconducting oxide grains to allow for isothermal grain alignment.

Results during the year include:

- The thermodynamic stability of $\mathrm{Bi}_{2} \mathrm{Sr}_{2} \mathrm{Ca}_{1} \mathrm{Cu}_{2} \mathrm{O}_{\mathrm{y}}$ thick films exposed to elevated oxygen fugacities $\left.\left[\mathrm{f}_{2} \mathrm{O}_{2}\right)=1-750 \mathrm{~atm}\right]$ and temperatures $\left(500-900^{\circ} \mathrm{C}\right)$ has been determined. Heat treatment at sufficiently high oxygen fugacities results in solid-state decomposition of the $\mathrm{Bi}_{2} \mathrm{Sr}_{2} \mathrm{Ca}_{1} \mathrm{Cu}_{2} \mathrm{O}_{\mathrm{y}}$-type compound into the $\mathrm{Bi}_{2}(\mathrm{Sr}, \mathrm{Ca})_{2} \mathrm{Cu}_{1} \mathrm{O}_{y}$-type compound, an alkaline earth bismuthate $\left(\approx \mathrm{BigSr}_{11} \mathrm{Ca}_{5} \mathrm{O}\right)$, and $\mathrm{CuO}$. The temperature at which such decomposition occurs decreases with increasing oxygen fugacity (e.g., $<500^{\circ} \mathrm{C}$ at $\mathrm{f}\left(\mathrm{O}_{2}\right)=750 \mathrm{~atm}$ ).

- An enhanced degree of grain alignment has been observed in $\mathrm{Bi}_{2} \mathrm{Sr}_{2} \mathrm{Ca}_{1} \mathrm{Cu}_{2} \mathrm{O}_{\mathrm{y}}$ thick films that have been decomposed and then re-formed by a two-stage, solid-state heat treatment consisting of:

- a 5 hour anneal at $880^{\circ} \mathrm{C}$ at an oxygen fugacity of $115 \mathrm{~atm}$. followed by

- a 24 hour anneal at $860^{\circ} \mathrm{C}$ in pure oxygen at 1 atm pressure. The resulting $\mathrm{Bi}_{2} \mathrm{Sr}_{2} \mathrm{Ca}_{1} \mathrm{Cu}_{2} \mathrm{O}_{\mathrm{y}}$ films were also more dense than films that were exposed to a single heat treatment at $860^{\circ} \mathrm{C}$ in $1 \mathrm{~atm}$ oxygen for up to 120 hours. 
- Bulk (Pb,Bi) $)_{2} \mathrm{Sr}_{2} \mathrm{Ca}_{2} \mathrm{Cu}_{3} \mathrm{O}_{10 \pm y}$-type specimens (produced from nitrate-pyrolyzed powder provided by American Superconductor Corporation) have also been found to undergo solid-state decomposition at elevated oxygen pressures. The decomposition reaction for this compound is still being determined.

\section{Pair Breaking Phenomena in the La2-xSrx Cu1-yNiy $\mathrm{O}_{4}$ Systems:}

\section{Experiment and Theory \\ J. M. Honig and J. Spalek \\ Purdue University}

The two questions being addressed by this project are:

1. What is the influence on $T_{c}$ of $\mathrm{Ni}$ substitutions for $\mathrm{Cu}$ as a function of the $\mathrm{Sr}$ concentration in $\mathrm{La}_{2-x} \mathrm{Sr}_{x} \mathrm{Cu}_{1-y} \mathrm{Ni}_{y} \mathrm{O}_{4+\delta}$ ?

2. What are the electron states and real space pairing of a statistical spin liquid (SSL) in which double occupancies of the same quasiparticle level are not allowed?

We have investigated the properties of an electron assembly in solids in which the charge carrier interactions are so strong that the double occupancy of states, usually considered in setting up the statistics of the standard electron gas, is no longer possible. A totally new theoretical treatment of the strongly interacting system has been set up and a.pplied to high $\mathrm{T}_{\mathrm{C}}$ superconductors. A rationalization has been provided of the experimental trends showing not only how $\mathrm{T}_{\mathrm{c}}$ changes with doping of these superconductors but also how the superconducting gap changes with temperature. Both sets of predictions are in very good accord with experiment.

Together with a second group at the University of Kentucky we have initiated a program of studies concerning the heat capacity anomalies at the onset of superconductivity in La2- $\mathrm{Sr}_{\mathrm{x}} \mathrm{Cu}_{1-\mathrm{yNi}} \mathrm{NO}_{4}$. The substitution of nickel for copper in the parent compound leads to a drastic decrease in the critical temperature, $\mathrm{T}_{\mathrm{c}}$. 


\section{Processing and Texture Development in $\mathrm{YBa}_{2} \mathrm{Cu}_{3} \mathrm{O}_{7-\delta} \delta$ Films Synthesized from Metallo-Organic Precursors \\ Gerald L. Liedl and Arden L. Bement, Jr. Purdue University}

The objectives of this research project are:

1. To achieve rapid thermal processing of $\mathrm{YBa}_{2} \mathrm{Cu}_{3} \mathrm{O}_{7-\delta}(\mathrm{YBCO})$ films on suitable substrates employing metallo-organic precursors; and

2. To understand and control the development of film orientation by varying the process parameters.

The Metallo-Organic Decomposition (MOD) technique was utilized in the fabrication of YBCO films with preferred orientation on single crystal (100) $\mathrm{MgO}$ and (100) $\mathrm{SrTIO}_{3}$ substrates. Rapid thermally processed Y-rich films $\left(870-980^{\circ} \mathrm{C}\right)$ exhibited a metallic behavior in the normal state with superconducting onset between $80-85 \mathrm{~K}$ and maximum orthorhombic peak splitting.

For films deposited on (100) MgO substrates, c-axis texturing is observed in all films fired above $930^{\circ} \mathrm{C}$ in an oxygen atmosphere. At higher temperatures $\left(\geq 945^{\circ} \mathrm{C}\right)$ there is a tendency for in-plane preferential orientation in addition to the c-axis texture. Epitaxial film growth has been observed in films fired at $2960^{\circ} \mathrm{C}$ in reduced oxygen pressures.

For films deposited on (100) $\mathrm{SrTiO}_{3}$ substrates, strontium interdiffusion and second phase formation (complex barium titanium oxide) is observed at firing temperatures $>940^{\circ} \mathrm{C}$ in an oxygen atmosphere. Epitaxial films, free of secondary phases, have been obtained by firing at $930^{\circ} \mathrm{C}$ in reduced oxygen pressure. 
High Temperature Superconducting Tubes for Magnetic Shielding Applications

K. P. Trumble, M. W. McElfresh, and K. J. Bowman

Purdue University

guantum Magnetics*

Los Alamos National Laboratory"

The objectives of this research project are:

1. To develop processes for fabricating superconductivity shield tubes;

2. To characterize the texture and properties of the processed tubes; and

3. To investigate the effects of annealing conditions on the superconducting properties of BSCCO-silver composites.

Results during the year include:

- BSCCO tubes processed by centrifugal slip casting have been produced consistently. The slurries used in centrifugal slip casting of these tubes have been optimized.

- Microstructure characterization using optical microscopy shows the higher alignment of BSCCO particles in centrifugally slip casting of tubes (five times that of a random sample) compared to that achieved by cold-pressing ( $50 \mathrm{MPa}$ ) the same powder. Compared to the coldpressed sample, centrifugal slip casting produces 15 to $20 \%$ higher fired density and $30 \%$ higher $\mathrm{J}_{c}$.

- We have recently shown that the vortex-glass phase transition of the grain boundary is significantly reduced with respect to that of the bulk. This reduction may be a result of oxygen depletion, which we also recently showed will push the vortex-glass transition to lower temperature and fields. The field dependence of the weak-link behavior is consistent with these results. 


\section{Study of Naturally Modulated High $T_{c}$ Superconducting Compounds}

\section{H. Sato}

\section{Purdue University}

The objective of this project is to use controlled changes in the natural crystal structure modulation of the high-temperature superconductors to understand superconductivity and guide improvements of materials.

In order to answer the question "Why in Bi-compounds are reliable data limited to the right hand side of the universal curve?" a paper "Structural Stability of Bi-based Cuprates" has been written. The conclusion is that $\mathrm{Bi}$-based compounds are not structurally stable without doping of oxygen or lead atoms in the $\mathrm{Bi}-\mathrm{O}$ layer and, hence, are only stable with sufficient concentration of $O$ ions.

Bi-compounds consist of the Perovskite block, which includes the $\mathrm{CuO}_{2}$ layer and the Rocksalt block representing the $\mathrm{Bi}-\mathrm{O}$ layer. If the mismatch of these two types of blocks becomes too large, the crystal becomes unstable.

For the measure of the stability of the Perovskite structure, the tolerance factor, $t$, is generally used. This is defined for a Perovskite $\mathrm{ABO}_{3}$ as

$$
t=\frac{A-O}{\sqrt{2(B-O)}}
$$

where the bond length A-O and B-O are taken to be the sum of the ionic radii of cation and oxygen. In perovskite, the structure is stable if $0.8<\mathrm{t}<0.9$. In $\mathrm{BI}$-compounds $\mathrm{Bi}$ is taken for " $\mathrm{A}$ " and $\mathrm{Cu}$ is taken for " $\mathrm{B}$ ". Even for Bi-compounds, $t=0.80$ is found to be the limit for the stability. Without extra oxygen doping in the Bi-O layer, $t$ is found to be -0.78 . Extra oxygen atoms increase the Bi-O bond length. Based on this, without the doping of $\mathrm{Pb}$, the minimum amount of extra oxygen required for stabilizing the $n=2$ compound or to make $t \geq 0.80$ is estimated to be $\delta_{\min } \approx 0.08-0.1$. It is apparent from this stability relation, that the existence of Bicompounds is limited to the right hand side of the universal relation. The result also shows clearly that the mismatch of the Rocksalt block and the Perovskite block is key to determine the stability of this compound. Here, 
the charge reservoir $\mathrm{BI}-\mathrm{O}$ layer is found to provide all extra charges to the superconducting $\mathrm{CuO}_{2}$ layers created by nonstoichiometry. 
Area 2: High Tc Superconductor Transport Properties: Controlling/Limiting Factors

Guantum and Statistical Mechanics of High $T_{c}$ Superconductors and Flux Line Liquids

\section{A. H. MacDonald and S. M. Girvin \\ Indiana University}

The objectives of this project are:

1. To clarify how superconductivity coexists with very intense magnetic fields at low temperatures in the limit when the effects of Landau quantization becomes important;

2. To investigate the quantum phase diagram of superconductivity in two-dimensional systems (e.g., thin films) in the presence of magnetic fields and/or disorder; and

3. To numerically simulate how columnar defects pin vortices in the high $T_{c}$ superconductors.

Results during the year include:

- We have delineated the phase diagram describing the transition region between normal and Abrikosov lattice states of anisotropic superconductors in a magnetic field by performing Monte Carlo simulations of the Lawrence-Doniach model.

- We have developed a formalism which makes fully microscopic calculations of mixed state properties possible for the first time and are applying it to calculations of the microwave conductivity.

- We have, for the first time, solved microscopic models for the magnetic field dependence of the upper critical field in the case of non s-wave superconductors. 
- We have performed Monte Carlo calculations which establish that the superconducting vortex glass state does not occur at finite temperature in the limit of $2 \mathrm{D}$ films with point disorder.

\section{Thermally Activated Flux Motion and Anisotropy in High-Temperature} Superconductors

J. R. Clem and V. G. Kogan

Iowa State University

AT\&T Bell Labs*

The objective of this project is to develop a quantitative theory of the relationship between the anisotropy of high-temperature superconductors and their susceptibility to thermally-activated fluxuation and other possible dissipative processes, in order to set theoretical limits on their critical current densities.

Starting with the theory of thermal fluctuations of vortices in layered superconductors, which we had developed during the previous year with MISCON support, we were able to analyze the magnetization data on $\mathrm{Bi}_{2} \mathrm{Sr}_{2} \mathrm{CaCu}_{2} \mathrm{O}_{\mathbf{x}}$ in order to extract some key superconducting characteristics of this material, including the temperature dependencies of the penetration depth, $\lambda_{a b}$, the upper critical field, $H_{c 2}$, and the GinzburgLandau parameter, $\boldsymbol{k}$.

If fluctuations are ignored, use of the mean-field theory to analyze the data yields a puzzling, unphysical increase of $\mathrm{H}_{\mathrm{C} 2}(\mathrm{~T})$ as $\mathrm{T}$ approaches $\mathrm{T}_{\mathrm{C}}$. Including fluctuations in the theory, however, resolves this problem and leads to a more reasonable temperature dependence for the experimentally-determined values of $\mathrm{H}_{\mathrm{C} 2}(\mathrm{~T})$ near $\mathrm{T}_{\mathrm{C}}$.

\section{Electrical and Optical Inwestigations of Pristine and Doped $\mathrm{YBa}_{2} \mathrm{Cu}_{4} \mathrm{O}_{8}$ Meera Chandrasekhar University of Missouri-Columbia}

The objective of this project is to study the role of $\mathrm{Zn}$ and Fe doping in $\mathrm{YBa}_{2} \mathrm{Cu}_{4} \mathrm{O}_{8}$ (Y124). The first experiment is to study the softening of the in-plane phonon modes at $T_{C}$ via Raman scattering for different dopant 
concentrations of $\mathrm{Fe}$ and $\mathrm{Zn}$, thereby establishing whether the effect of the weak links is a minimum at a critical nominal concentration, as in Y123.

Results during the year include:

- A study of the phonon modes in $\mathrm{YBa}_{2} \mathrm{Cu}_{4} \mathrm{O}_{8}$ (Y124) was conducted using Raman scattering under hydrostatic pressure (up tolo0 kbar) at 13K. These are the first studies of these materials under pressure. We have accurately determined and compared the mode Grüneisen parameters for six different phonon modes, including those associated with various vibrations either in the $\mathrm{Cu}-\mathrm{O}$ planes or in the Cu-O chains. A marked difference can be observed $b$ tween the behavior of these chain and plane modes. The mode Grüneisen parameters of the phonons present in both the $\mathrm{YBa}_{2} \mathrm{Cu}_{4} \mathrm{O}_{8}$ and the more conventional superconductor $\mathrm{YBa}_{2} \mathrm{Cu}_{3} \mathrm{O}_{7}(\mathrm{Y} 123)$ scale with their bulk moduli, while the chain modes that are Raman allowed only in $\mathrm{YBa}_{2} \mathrm{Cu}_{4} \mathrm{O}_{8}$ are anomalously different.

- A study of the phonon anomalies in pristine and Zn-doped Y124 was conducted at atmospheric pressure. In Y123 materials the frequencies of the in-plane phonon modes exhibited a softening at $\mathrm{T}_{\mathbf{c}}$ that is attributed to mixing with electronic excitations across the superconducting gap. In Y124 materials, the several phonons (not just the in-plane) exhibit such anomalies.

- A study of the shifts in the phonon modes was conducted in Y124. The high-frequency, chain-associated mode shifts very rapidly with pressure, while the other modes shift to lower frequencies. The Raman-frequency shifts in most materials are usually small and extend to higher frequencies. As such, this behavior in Y124 is rather unusual. 


\section{Neutron Diffraction and Defect Studies of High $T_{c}$ Superconductors \\ W. B. Yelon and J. W. Farmer \\ University of Missouri-Columbia}

The major current objective of this project is to model current-voltage characteristics, critical currents, phase boundaries, and other properties in granular and otherwise disordered high-Tc superconductors, principally using networks of Josephson junctions.

Results during the year include:

- In collaboration with Ames Lab, we have developed a better understanding of the behavior of Nd-rich 1-2-3 compounds

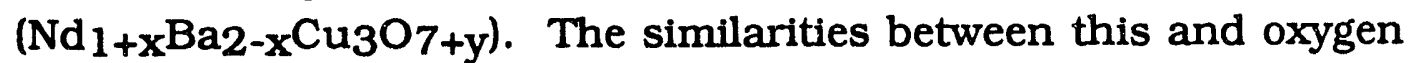
deficient Y123 are related to the addition of excess oxygen on the anti-chain sites and the filling of holes.

- The collaboration with S.K. Malik has resulted in three publications on related Pr compounds, which, while non-superconducting, may be useful in making practical devices and in improving the critical current density. A new structure has been found, and work is underway to prepare further related compounds both with $\mathrm{Pr}$ and other rare earths.

- An important new collaboration has begun with the University of Kansas and Midwest Superconductivity, Inc. (Lawrence KS) to characterize Tl-based compounds. This group is preparing devices (especially thin films and bulk shielding) through the use of a novel synthesis route which gives better material than conventional processes.

- We developed a new, direct method for analyzing magnetic relaxation data. This method gives both the slope and second derivative of the pinning potential, $\mathrm{U}(\mathrm{m})$. With the new method it is possible to map $\mathrm{U}(\mathrm{m})$ in detail and determine the dependence of $U(\mathrm{~m})$ on $B$, the 
magnetic induction, as well as to clearly see the effects of radiationinduced pinning centers on $\mathrm{U}(\mathrm{m})$.

- We have proposed a simple, one parameter model of the dependence of the critical current on B. The model accounts for the qualitative shape of hysteresis curves in both weak and strong pinning systems and can be used to explain the change of shape of hysteresis curves after the introduction of pinning centers with irradiation.

\section{Microwave and Optical Studies of High-Temperature Superconductors: \\ Flux-Lattice Dynamics, Crystal Anisotropy, Flux Pinning and Photo- Modulated Superconductivity \\ H. A. Blackstead \\ University of Notre Dame}

This project studies transport properties of high-temperature superconductors, in particular field-induced changes in the microwave resistance, to derive information on flux lattice dynamics, crystal anisotropy and flux pinning.

We have recently concluded field and temperature-dependent, surface resistance measurements on superconducting, polycrystalline, Hg$\mathrm{Ba}-\mathrm{Ca}-\mathrm{Cu}-\mathrm{O}$. We found that several samples provided to us contained multiple phases, including primarily $\mathrm{HgBa}_{2} \mathrm{CaCu}_{2} \mathrm{O}_{6}+\delta$ (two layer) and $\mathrm{HgBa}_{2} \mathrm{Ca}_{2} \mathrm{Cu}_{3} \mathrm{O}_{8}+\delta$ (three layer) phases. A small amount of a third phase, most likely $\mathrm{HgBa}_{2} \mathrm{Ca}_{3} \mathrm{Cu}_{4} \mathrm{O}_{10}+\delta$, was also detected. With variation of the angle $\Psi$ between the applied field and current density, the low temperature data exhibit large, flux-flow resistivity which varies approximately as $\sin ^{2}(\Psi)+a(B)$. For low temperatures, $a(B)$ is a relatively small isotropic field dependent contribution. At high temperatures, the dominant field and temperature dependent response is attributed to the isotropic phase-slip resistivity losses in intra-granular defects. For temperatures just below the transition temperature of the 2-layer phase, very small flux-flow is evidenced. This result is consistent with a large effective $\mathrm{H}_{\mathrm{c} 2}$, suggesting a "dirty-limit" value of $\mathrm{H}_{\mathrm{c} 2}$. The interesting features of the data required significant revisions of applicable theory. This 
has led to the development of a theory which merges the simpler approach we have previously employed, with the sophisticated Coffey-Clem theory.

\title{
Superconducting Quantum Structures
}

\author{
A. E. Miller, B. Das, and \\ S. Bandyopadhyay \\ University of Notre Dame
}

The objective of this project is to fabricate lateral surface superlattices (two dimensional periodic arrays of quantum dots) of the maximum temperature superconductor BKBO.

The fabrication requires the generation of a two dimensional template that acts as a host for the quantum dots. This template is a regular, periodic hexagonal arrangement of ultrasmall pores that form in the anodized layer on $\mathrm{Al}$.

We have successfully completed the first phase of this research, namely setting up the anodizing equipment with the necessary controls. Several test runs have been made to standardize the anodization process. Anodized $\mathrm{Al}$ has been produced that show the desired pore formation.

We are now poised to enter the second phase which is backfilling the pores with the superconducting materials of interest.

\section{Transport Properties of YBCO Thin Films \\ S. T. Ruggiero \\ University of Notre Dame}

The objective of this project is to understand and control the electronic properties of YBCO thin films; in particular:

1. To study critical-current enhancement by induced-defect generation, and

2. To investigate the far-infrared responsivity of step-edge YBCO devices. 
Results during the year include:

- We have successfully reconfigured our deposition system to focus our effort, with an emphasis on YBCO film growth and step etching. We have verified the ability to produce good thin films, now far more efficiently, with step etching in the same chamber.

- FIR diode laser tests are proceeding with one laser in hand. Our completed system will have the ability to do both unique mixing (at $25 \mu \mathrm{m} !)$ and time-domain ( $<300$ ps) experiments.

\section{Far-Infrared and Microwave Magneto-Dissipation in High-Tc \\ Superconductors \\ W. J. Tomasch and J. K. Furdyna \\ University of Notre Dame \\ NIST* \\ TRW Superconductivity Research*}

The objectives of this research project are:

1. To study the dynamic properties of the flux lattice in highly anisotropic high temperature superconductors; and

2. To utilize laser and microwave magneto reflection and transmission measurements to obtain quantitative determinations of the important material parameters that are of basic interest and appropriate for high frequency applications.

Results during the year include:

- We have developed a leadless, nondestructive microwave technique for quantitative evaluation of high temperature superconductors in the presence of substantial dc magnetic fields (0-7 tesla). Recent improvements enable us to infer the microwave surface impedance directly from the data. This avoids an intermediate step involving use of some specific theory of the mixed state. We are now able to numerically solve Maxwell's equations for the electromagnetic field 
distributions in the sample chamber. This is a potentially important factor impacting our accurate determination of the surface impedance.

- Analysis of magneto transmission and reflection measurements on a 1800 angstrom thick YBCO (123) film, supplied by TRW, Inc., have yielded realistic material parameters.

\section{High $T_{c}$ Superconducting Composites, Arrays and Crystals J. C. Garland The Ohio State University}

The objectives of this project are:

1. To develop high-T $T_{C}$ machinable $A g / Y B C O$ bulk composites with superconducting fractions ranging from 10 percent to 100 percent;

2. To develop high-T $T_{c} A g / Y B C O$ weak links and fabricate twodimensional (2-D) Josephson Junction arrays; and

3. To prepare BSCCO single crystals and understand the effects of interplane vortex interactions.

Results during the year include:

- We have developed machinable bulk superconducting $\mathrm{Ag} / \mathrm{YBa}_{2} \mathrm{Cu}_{3} \mathrm{O}_{7}$ composites whose resistive transition temperatures were those of pure $\mathrm{YBa}_{2} \mathrm{Cu}_{3} \mathrm{O}_{7}$. By systematically varying the superconductor volume fraction over the entire percolation range $(0.16-0.9)$, several high- $T_{c}$ composite materials were synthesized for low-current applications, including superconducting shielding enclosures, high- $Q$ tuned cavities and bonding materials.

- We have fabricated and characterized $\mathrm{Ag} / \mathrm{YBa}_{2} \mathrm{Cu}_{3} \mathrm{O}_{7}$ superconducting weak link junctions and arrays on $\mathrm{SrTiO}_{3}$ substrates. Each weak link junction consisted of a $5 \mu \mathrm{m}$-wide high$\mathrm{T}_{\mathrm{C}}$ film overlaying at right angles to a $0.5 \mu \mathrm{m}$-wide silver line. 
Measurements of the zero-field resistive transitions and currentvoltage characteristics showed that our two-dimensional arrays, which contained $300 \times 300$ proximity-coupled weak link junctions, exhibited a Kosterlitz-Thouless-type transition to the superconducting state.

- Our six-contact-lead measurements of the zero-field resistive transition of $\mathrm{Bi}_{2} \mathrm{Sr}_{2} \mathrm{CaCu}_{2} \mathrm{O}_{8}$ single crystals showed that, with decreasing temperature, a bulk zero-resistance state is established via Josephson coupling of the $\mathrm{CuO}$ bilayers, followed by a KosterlitzThouless-type transition in the bilayers. In the temperature interval $(\sim 2 \mathrm{~K})$ bounded by the two transition temperatures, our "fluxtransformer" experiments yielded a pronounced peak in the secondary voltage, which was suppressed by external magnetic fields. We associate this structure with the out-of-plane Josephson coupling of thermally-excited vortices and antivortices.

\section{Atomic Beam Studies of Vortex Lattices at Superconductor Surfaces Gregory Lafyatis and Thomas Lemberger The Ohio State University}

The long-term goals of this work is to measure vortex correlation functions at the surface of superconductors by developing a new atomic beam technique and to use this technique to study the behavior of the vortex lattice in high- $T_{c}$ materials.

Results during the year include:

- In the late spring (May 10) we accomplished our first benchmark goal for this project: we saw a vortex lattice in a sputtered superconducting niobium film.

- In analyzing and modelling our results, we discovered that our signal strength is sensitive to the penetration depth of the sample and that by examining our signal strength as a function of temperature. 
(surprisingly) we could determine the penetration depth of our samples.

- The most important improvement in the past half-year is our description of the individual vortices. We now use the London model description of Marchettl, extended to include the finite thickness of our sample.

- The power and potential of this new technique depend on our signal detection sensitivity. By cooling an avalanche photodiode down to liquid nitrogen temperature we are able to achieve a sensitivity between a factor of two and a factor of one hundred better than the best devices that are currently on the market. For our experiment, it should soon result in a factor of thirty increase in sensitivity.

Device-Related Properties of Doped $\mathrm{YBa}_{2}\left(\mathrm{Cu}_{1-x} \mathrm{M}_{x}\right)_{3} \mathrm{O}_{7-\delta}$ Films

Thomas R. Lemberger The Ohio State University

The objective of this project is to develop a better understanding of transport properties of superconductors through measurements of the kinetic inductance and Bernoulli potential in YBCO films.

Results during the year include:

-We demonstrated that the size and temperature dependence of the magnetic penetration depth measured in films of $\mathrm{YBa}_{2} \mathrm{Cu}_{3} \mathrm{O}_{7-\delta}$ agrees in large measure with earlier surprising and controversial measurements on crystals. Differences between our films and crystals are quantitatively explained as resulting from weak disorder in a $d$-wave superconductor, thus supporting the conclusion from the crystal work that pure $\mathrm{YBCO}$ is a d-wave superconductor.

- We demonstrated that oxygen depletion from pure YBCO films increases the magnetic penetration depth without affecting its dependence on $T / T_{c}$. This is consistent with $d$-wave 
superconductivity and the popular opinion that oxygen depletion changes the concentration of superconducting charge carriers without adding substantial disorder to the important CuO plane bilayers.

- We demonstrated that replacing a few percent of $\mathrm{Cu}$ with $\mathrm{NI}$ or $\mathrm{Zn}$ in YBCO films increases the penetration depth dramatically and alters its dependence on temperature. These effects are consistent with estimates for the behavior of $d$-wave superconductors, assuming that doping introduces disorder without affecting the concentration of superconducting carriers.

\section{NMR Studies of Vortex Dynamics in High $T_{c}$ Superconductors Charles Pennington The Ohio State University}

The objective of this project is to make NMR measurements on several high $T_{c}$ samples with the goal of finding situations favorable to the detection and characterization of vortex motion.

We are investigating several types of high $T_{c}$ materials, using several nuclear species as probes. Highlights of our research on the use of NMR to explore vortex dynamics in high $T_{C}$ superconductors are:

- We measured 205TI NMR linewidths in single crystal 2201 and observed indications of possible decoupling of vortices in adjacent $\mathrm{CuO}_{2}$ planes.

- We used $89 \mathrm{Y}$ 2D NMR on $\mathrm{YBa}_{2} \mathrm{Cu}_{3} \mathrm{O}_{7}$ powder to demonstrate that at 9 Telsa and 20-90K the correlation time for vortex jumps of a size comparable to the vortex lattice spacing is much greater than $10 \mathrm{~ms}$.

- In 89Y NMR measurements of Powder $\mathrm{YBa}_{2} \mathrm{Cu}_{3} \mathrm{O}_{7}$ our experiments yield a simple conclusion; namely, that in fields of several Tesla, vortices do not make frequent spatial jumps of distances comparable to the lattice spacing. 
- In 205TI NMR measurements of 2201 Single Crystals anomolies in $205_{\mathrm{Tl}}$ line shapes at low magnetic fields suggest that vortices in adjacent plane become decoupled at higher temperatures. The position of a vortex current loop in one plane becomes completely uncorrelated with that of the vortex in an adjacent plane. As a result. the magnetic fields produced by vortex current loops in adjacent planes add in an incoherent matter, and NMR and $\mu$ SR linewidths are much narrower than would be expected.

\section{Dynamical Properties of Josephson-coupled Systems: Application to High- Temperature Superconductors \\ David G. Stroud The Ohio State University}

This project is intended to study the structures of various high $T_{C}$ superconductors, their defects, the correlations between their defects and physical properties, and the control of these defects.

During the past several months, we have succeeded in calculating the flux-lattice melting temperature of single-crystal $\mathrm{YBa}_{2} \mathrm{Cu}_{3} \mathrm{O}_{7}-\delta$ without any adjustable parameters, in excellent agreement with the experimental results of Safar, et al, over a fleld range from about $10^{4}$ to $1.5 \times 10^{5}$ Oe (HIlc).

Besides obtaining the flux lattice melting curve, we have begun to obtain some properties of the two phases near the transition. First, it appears that there is a single phase transition, characterized by the simultaneous onset of long-range order in both the c direction and the $a b$ plane.

Secondly, we find that the transition in the pure case is characterized by the onset of a finite superfluid density component in the c direction, but not in the ab plane. This corresponds, not to a flux line liquid, but rather to a flux lattice which is unpinned and free to slide in the ab plane. 


\section{Transport Properties of Figh $T_{c}$ Superconductors \\ G. F. Giuliand \\ Purdue University}

The objectives of this project are:

1. To advance the theory of Josephson junctions and the flux dynamics in Type-II superconductors; and

2. To characterize the transport properties of artificially manufactured Josephson junctions as a means of better understanding the Josephson effort in layered superconducting structures.

Our research efforts have led to a number of interesting and fundamental results:

- We have developed a theory of the effect of an applied magnetic field, $H_{\text {ext, }}$ on the critical current, $\mathrm{J}_{\mathrm{c}}$, of a bulk layered superconductor. We believe that our results provide an understanding for some of the interesting transport properties recently observed in high $T_{C}$ (bulk) cuprates.

- We have studied the effect of an applied magnetic field, Hext, on the critical current, $I_{c}$. of a single Josephson junction. The occurrence of an interesting cancellation phenomenon between the effects of the Meissner current and that associated with vortex currents has been demonstrated and analyzed in detall.

- We have studied the general problem of a Josephson junction with thin superconducting electrodes. In this case field penetration can occur in the form of Pearl vortices and the dependence $\mathrm{I}_{\mathrm{C}}\left(\mathrm{H}_{\mathrm{ext}}\right)$ can differ greatly from the usual "Fraunhofer" relationship characterizing homogeneous field penetration.

- We developed a theory of the current-voltage characteristic of a Josephson junction in the presence of randomly distributed Abrikosov vortices in the superconducting electrodes. We have found 
that for finite blas voltage, propagating electromagnetic waves display a broad spectrum of wavelengths. Moreover the amplitude of these waves is found to increase as the blas is decreased.

- We have calculated the Josephson current for a S-I-S junction taking into account the effect of the interaction between Cooper pairs and phonons in the insulating barrier region. We show that in the limit of $\omega \tau \mathrm{gg} 1$ (where $\omega$ is here a characteristic phonon frequency and $\tau$ is the semiclassical barrier crossing time) the critical current can drastically change. In particular we have established that when the screening length is much larger than the barrier thickness the critical current can actually increase with respect to the case of negligible barrier-electron-phonon interaction.

- We have investigated the spatial dependence of $v_{n}(r)$, the normal current in the vicinity of a moving vortex. This investigation provides insight into the appropriate boundary conditions to be used in a realistic modeling of the intermediate region which characterizes the immediate vicinity of a moving vortex core in a superfluid.

\section{Structural Defects and Interfaces in High $T_{c}$ Superconducting Compounds Eric P. Kuam \\ Purdue University}

The objectives of this project are:

1. To examine the microstructure, and in particular, the extended defect and intercrystalline boundary structures of textured bulk polycrystals and epitaxial thin films (including bicrystals) of $\mathrm{YBa}_{2} \mathrm{Cu}_{3} \mathrm{O}_{7}$; and

2. To correlate these microstructural aspects with transport property characteristics. 
In materials with 211 additions, it has been found that the enhancement of critical currents through flux pinning depends on the size of the 211 particles. The pinning seems to be induced by other extended defects (dislocations and CuO layer stacking faults) associated with the particles, rather than directly by the particles themselves.

Another aspect which has been identified is the existence of very small precipitates, apparently of the 211 phase. These precipitates are in the 30 to $100 \mathrm{~nm}$ size range, and thus may be associated with magnetic pinning of flux lines.

In the calculation of the flux pinning by dislocations in 123 materials, we have shown that screw components of dislocations should have no strain-related effects on the flux lines, although the edge components may be significant contributors to pinning. Pinning effects related to dislocation cores, on the other hand, appear to be the other way around, at least for the special case of spiral growth-related screw dislocations. Such dislocations should have very larger Burgers vectors, and consequently very large normal-state cores, in which the flux lines could be readily trapped.

Intrinsic and Induced Weak Link Behavior in $\mathrm{YBa}_{2} \mathrm{Cu}_{3} \mathrm{O}_{7-\delta} \delta$ Films

M. W. McElfresh and R. G. Reifenberger

Purdue University

IBM Thomas J. Watson Research Center*

Lake Shore Cryotronics*

Quantum Magnetics*

The Weirmann Institute of Science*

The objectives of this project are:

1. To fabricate and characterize high-temperature superconducting thin films; and

2. To optimize material properties through a better understanding of the order parameter and weak-link behavior in high-temperature superconductors. 
Results during the year include:

- We observed evidence for a two vortex-phase transitions in T1-2212. The lower temperature transition corresponds to a vortex-glass transition and the higher temperature transition to a pancakevortex-coupling transition.

- The magnetic phase diagram for grain boundaries in polycrystalline YBCO showed that the vortex-glass transition is strongly reduced at the boundary. Also, we observed a reduction in $\mathrm{T}_{C}$ at the grain boundary of a bicrystal film.

- We measured the magnetic phase diagram of $\mathrm{YBa}_{2} \mathrm{Cu}_{3} \mathrm{O}_{7-\delta} \delta$ as a function of $\delta$, showing that the vortex-phase transition moves down and broadens with $\delta$, while the melting transition does not broaden but merely tracks the change in $T_{C}$.

- We explained the anomalous reduction in magnetic-relaxation rate after a transport current is removed from a thin film and reported detailed analytical calculations of the demagnetization effects on thin film samples.

- We reported the frequency dependence of the vortex-glass transition by magnetotransport and showed that it is frequently independent for over five orders of magnitude in frequency.

- We identified the fine structure in the density of states data for YBaCuO high $\mathrm{T}_{\mathbf{c}}$ films. The fine structure observed compares favorably with a theoretical model proposed by Tachiki, et al.

- We observed a single electron tunneling behavior in layered, high $\mathrm{T}_{\mathrm{C}}$ $\mathrm{TlBa}_{2}\left(\mathrm{Ca} 0.8 \mathrm{Y}_{0.2}\right)_{\mathrm{Cu}} \mathrm{O} 7$ at $10 \mathrm{~K}$. By successfully applying a threetunnel capacitor model for single electron tunneling, we were able to explain the highly non-linear I-V characteristics obtained from $\mathrm{TlBa}_{2}\left(\mathrm{Ca0} .8 \mathrm{Y}_{0.2}\right)_{\mathrm{Cu}} \mathrm{O}_{7}$ films. 
- We realized that layered materials of the type commonly studied by MISCON make attractive materials for fabricating 1-D arrays of tunnel-capacitors. Such arrays are one essential building block for the next generation of nanoelectronic devices.

\section{Vortices in Anisotropic Superconductors Paul Mueilkar Purdue University}

The objectives of this project are to study vortex lattices and their instabilities in an effort to find minimum energy configurations and to study the pinning of a vortex line to a defect in a two-dimensional superconducting sheet.

Finding the configuration of vortex lines which minimizes the London free energy turns out to be a nontrivial task for anisotropic superconductors. We have found a surprising result: when the average field $\vec{B}$ is not parallel to a crystalline symmetry axds, a lattice of vortex lines parallel to $\vec{B}$ is not necessarily the configuration of lowest free energy. When the anisotropy is high enough, a configuration consisting of a set of vortex lines parallel to $\hat{c}$, plus a set of lines parallel to $\hat{a}$, and producing the same average $\vec{B}$, can have a lower free energy. We have made microscopic calculations of the penetration depth, $\lambda$, at $T=0$, as a function of impurity scattering; we find significant differences between $s$-wave and $d$-wave order parameters. 


\section{MISCON TALKS and PRESENTATIONS}

\section{January 1993 - December 1993}

1. Kenneth G. Caulton, Talk at West Virginia University, April 1993.

2. Malcolm H. Chisholm, Talk at the Gordon Research Conference on Chemical Reactions at Surfaces, Ventura, CA, March 1993.

3. "Numerical Simulations of Vortex Glass Dynamics", S. M. Girvin, Aspen Winter Meeting on Collective Dynamics in Disordered Systems, Aspen, CO, January 9, 1993.

4. "The Universal Conductivity at the Zero-Temperature SuperconductorInsulator Transition in Two Dimensions: Monte Carlo Calculation", Min-Chul Cha and S. M. Girvin, 2nd CTP Workshop on Statistical Physics, Seoul National University, Seoul, Korea, January 13-15, 1993.

5. "Quantum and Statistical Mechanics of High-Temperature Superconductors", S. M. Girvin, MISCON Meeting, Columbia, MO, January 28, 1993.

6. "Just What is Supercon ductivity Anyway?", S. M. Girvin, Indiana State University, Terre Haute, IN, March 17, 1993.

7. "I-V Characteristics of High Temperature Superconductors with Correlated Defects", Mats Wallin and S. M. Girvin, Proc. NATO ASI: Phase Transitions and Relaxation in Systems with Competing Energy Scales, Geilo, Norway, April 13-23, 1993.

8. "Vortex Glass Transition with Columnar Defects", S. M. Girvin, University of Minnesota, Minneapolis, MN, April 20, 1993.

9. "Universal Conductivity of a 2D Disordered Boson Model at the FieldTuned Superconductor-Insulator Transition", Min Chul Cha and S. M. Girvin, Bull. Am. Phys. Soc. 38, 526, 1993.

10. "Vortex Glass Transition with Columnar Defects", S. M. Girvin, University of Illinois, Urbana, IL, May 7, 1993.

11. "Vortex Glass Transition with Columnar Defects", S. M. Girvin, Stanford University, Stanford, CA, May 13, 1993.

12. "Quantum and Statistical Mechanics of Vortices", S. M. Girvin, MISCON Meeting, University of Notre Dame, South Bend, IN, July 7, 1993. 
13. "Just What is Superconductivity Anyway?", S. M. Girvin. University of Texas, Austin, TX, September 29, 1993.

14. "The Zero-Temperature Two-Dimensional Vortex Glass Transition", Ross A. Hyman, S. M. Girvin, Mats Wallin, A. Peter Young, and Matthew P. A. Fisher, 21st Midwest Solid State Theory Meeting, Detroit, MI, October 3-4, 1993.

15. "Just What is Superconductivity Anyway?", S. M. Girvin, University of Kansas, Lawrence, KS, October 25, 1993.

16. "Superconductors in Magnetic Fields", A. H. MacDonald, Atlantic Undergraduate Physics Conference, Antigonish Nova Scotia, February 1993.

17. "Superconductivity in Extremely Strong Magnetic Fields", A. H. MacDonald, University of Kentucky, April 1993.

18. "Superconductivity in Extremely Strong Magnetic Fields", A. H. MacDonald, University of Illinois, April 1993.

19. "Superconductors in Magnetic Fields", A. H. MacDonald, University of Louisville, April 1993.

20. "Vortex-lattice Melting", A. H. MacDonald, MISCON Workshop on Type-II Superconductors, Notre Dame University, July 1993.

21. "Two-Dimensional Vortex Lattice Melting", Jun Hu and A. H. MacDonald, Conference on the Statics and Dynamics of Vortices, Eugene, OR, August 1993.

22. "Vortex-lattice Melting in 2D Superconductors", A. H. MacDonald, Florida State University, October 1993.

23. "Vortex-lattice Melting in 2D Superconductors", A. H. MacDonald, University, of Florida, October 1993.

24. "Thermal Fluctuations and Flux Lattice Melting of Superconducting Films", Jun Hu and A. H. MacDonald, Bull. Am. Phys. Soc, 28, 635, 1993.

25. "Vortices in Layered Superconductors, I and II", John R. Clem, Experimental Workshop on High-Temperature Superconductors and Related Materials, Centro Atomico Bariloche, San Carlos de Bariloche, Argentina, January 11-12, 1993. 
26. "Fundamentals of Vortex Structure in High-Temperature Superconductors", John R. Clem, SC Global 93-International Superconductor Applications Convention, San Jose, CA, February 2, 1993.

27. "The Layered High-Temperature Superconductors: Why Is Their Ability to Carry Supercurrent Limited by Quasi-twodimensionality?", John R. Clem, Physics Colloquium, University of California, Davis, CA, February 23, 1993.

28. "Effects of Anisotropy and Layering in the High-Temperature Superconductors", John R. Clem, IVth International Bar-Ilan Conference on Frontiers in Condensed Matter Physics, Bar-Ilan University, Ramat-Gan, Israel, March 18, 1993.

29. "Vortex Structure of the High-Temperature Superconductors: 2D Pancake Vortices and Josephson Strings", John R. Clem. Berkeley, CA, March 31, 1993.

30. "Vortex Structure of the High-Temperature Superconductors: 2D Pancake Vortices and Josephson Strings", John R. Clem. Condensed Matter Physics Seminar, Oregon State University, Corvallis, OR, April 28, 1993.

31. "High-Temperature Superconductivity Update", John R. Clem, Annual Advisory Review of Exploratory Research, Electric Power Research Institute, Palo Alto, CA, May 5, 1993.

32. "Theory of ac Losses in Type-II Superconductors, Including the HighTemperature Superconductors", John R. Clem, EPRI/ASC Workshop on Bismuth Cuprate Superconducting Wires and Tapes, Half Moon Bay, CA, May 19, 1993.

33. "Vortex Structure of the High-Temperature Superconductors: 2D Pancake Vortices and Josephson Strings", John R. Clem, Condensed Matter Physics Seminar, University of California, Los Angeles, CA, June 9, 1993.

34. "Vortex Structure of the High-Temperature Superconductors: 2D Pancake Vortices and Josephson Strings", John R. Clem, Conductus, Sunnyvale, CA, June 17, 1993.

35. "Vortices in Highly Anisotropic Superconductors", John R. Clem, Tutorial Lecture, Midwest Superconductivity Consortium, Notre Dame, IN, July 9, 1993. 
36. "Anisotropic Superconductors, Fundamentals of Vortices in Layered Superconductors: I, II, III, and IV", John R. Clem, Tutorial lectures, NATO Advanced Study Institute on Vortices in Superfluids, Cargese, Corsica, France, July 21-22, 1993.

37. "2D Pancake Vortices in a Finite Stack of Superconducting Layers", John R. Clem, Euroconference on Coherence and Phase Transitions in Superconductors and Mesoscopin Structures, Institute for Scientific Interchange, Villa Gualino, Torino, Italy, September 28, 1993.

38. "Conference Summary", John R. Clem, Euroconference on Coherence and Phase Transitions in Superconductors and Mesoscopic Structures, Institute for Scientific Interchange, Villa Gualino, Torino, Italy, September 29, 1993.

39. "Entropy Effects in the Mixed State of Layered Superconductors", Vladimir G. Kogan, 2nd International Conference on High-Tc Superconductivity, Eilat, Israel, January 4, 1993.

40. "Fluctuations of Vortices in Weakly Coupled Layered Superconductors", Vladimir G. Kogan, AT\&T Bell Labs, Murray Hill, NJ, February 19, 1993.

41. "Role of Vortex Fluctuations in Determination of Penetration Depth from Magnetization for Layered Superconductors". J. H. Cho, D. C. Johnston, v. G. Kogan, M. Ledvij, and A. Yu. Simonov, APS March Meeting, Seattle, WA, March 23, 1993.

42. "Layered High- $T_{c}$ Superconductors in an ac Field", A. Yu. Simonov, APS March Meeting, Seattle, WA, March 23, 1993. (Clem, Kogan Group)

43. "Low Temperature Fluctuations of Vortices in Layered Superconductors", M. Ledvij, V. G. Kogan, Y. -Q. Song, W. P. Halperin, and L. N. Bulaevskii, APS March Meeting, Seattle, WA, March 25, 1993.

44. "Images of the Vortex Lattice Near Extended Twin Boundaries in YBa2 $\mathrm{Cu}_{3} \mathrm{O} 7-\delta "$ " P. L. Gammel, C. A. Duran, D. J. Bishop, V. G. Kogan, M. Ledvij, and A. Yu. Simonov, APS March Meeting, Seattle, WA, March 25, 1993.

45. "Fluctuations of Vortices in Josephson-Coupled Layered Superconductors", Vladimir G. Kogan, APS March Meeting, Seattle, WA, March 26, 1993. 
46. "Vortices in Superconductors", Vladimir G. Kogan, Summer School on Vortices in the Four States of Matter, Institute for Nonlinear Studies, UCSD, LaJolla, CA, June 21-23, 1993.

47. "Vortex Entropy in Layered Superconductors", Vladimir G. Kogan, Tutorial Lecture, Midwest Superconductivity Consortium Workshop, Notre Dame, IN, July 9, 1993.

48. "Vortex Interactions and the Flux-line Lattice", Vladimir G. Kogan, Workshop on Statics and Dynamics of Vortices in Superconductors, Eugene, OR, August 3, 1993.

49. "Hydrostatic Pressure Studies of the LO Phonon Modes in the Raman Spectra of the Bulk High-Temperature Superconductor $\mathrm{YBa}_{2} \mathrm{Cu}_{4} \mathrm{O}_{8}$ and the Effects of Doping", M. S. Boley, Meera Chandrasekhar, H. R. Chandrasekhar, Y. Wu, and P. Boolchand, AIRAPT/APS Conference, June 28-July 2, 1993.

50. "Raman Scattering Studies of Yb-124 Single Crystals and Y-124 Superconductor Samples under High Pressure", D. J. Payne, E. M. Baugher, R. J. Thomas, M. S. Boley, M. Chandrasekhar, H. R. Chandrasekhar, B. Dabrowski, Y. Wu, and P. Boolchand, Midwest Solid State Conference, September 24-25, 1993.

51. "High Pressure Optical Spectroscopy in Superconductors and Semiconductors", M. Chandrasekhar, Condensed Matter Seminar, Washington University, St. Louis, September 28, 1993.

52. "Critical Current Enhancement by Neutron Irradiation of Rapidly Textured $\mathrm{Bi}_{2} \mathrm{Sr}_{2} \mathrm{CaCu}_{2} \mathrm{O} 8 "$, M. J. Kramer, J. W. Farmer, S. R. Arrasmith, and R. W. McCallum, IEEE Trans. on Superconductivity, 1993.

53. "Magnetic and Heat Capacity Measurements on $\mathrm{Zn}$ - and Ga- doped PrBa2Cu3O7-8", S. K. Malik, H. Jhans, S. K. Dhar, w. B. Yelon, J. J. Rhyne, Ram Prasad, and N. C. Soni, Conference on Low Temperature Physics, 1993.

54. "Structural and Magnetic Properties of $\mathrm{PrBa}_{2} \mathrm{Cu}_{3} \mathrm{O}_{\mathrm{x}}(\mathrm{x}=6.9$ and 6.4)", S. K. Malik, W. B. Yelon, J. J. Rhyne, Ram Prasad, and N. C. Soni, Physics \& Chemistry of Molecular \& Oxides Superconductors, Eugene, Oregon, July 27-31, 1993.

55. "Anisotropic Resistivity in Y123 Films and Crystals", H. A. Blackstead, March APS Meeting, Seattle, WA, March 1993. 
56. "Field and Temperature Dependent Surface Resistance in High Temperature Superconductors", H. A. Blackstead, M. I. T. Lincoln Lab, November 5, 1993.

57. "Growth Morphologies of [001], [100] and [010] Oriented

$\mathrm{Er}_{5} \mathrm{Ba}_{7} \mathrm{Cu}_{12} \mathrm{O}_{\mathrm{y}}$ High-Temperature Superconductor Thin Films on Various Substrates", K. M. Choudhary, P. Seshadri, and M. Black, Thirteenth North American Molecular Beam Epitaxy Conference, Stanford, CA, September 13-15, 1993.

58. "Surface Morphology of $\mathrm{Dy}_{1} \mathrm{Ba}_{2} \mathrm{Cu}_{3} \mathrm{O}_{7-} \delta$ and $\mathrm{Y}_{1} \mathrm{Ba}_{2} \mathrm{Cu}_{3} \mathrm{O}_{7-} \delta$ Thin Films", J. Bae, P. Seshadri, T. Ekkens, M. Honkannen, C. Zhong, S. T. Ruggiero, and K. M. Choudhary, Bull. Amer. Phys. Soc. 37, 333 (1993).

59. "The Effect of $\mathrm{PtO}_{2} \times \mathrm{H}_{2} \mathrm{O}$ Additions on the $\mathrm{Y}_{2} \mathrm{BaCuO}_{5}$ Morphology in Melt Textured $\mathrm{YBa}_{2} \mathrm{Cu}_{3} \mathrm{O}_{7-\mathrm{x}}$., C. Varanasi and P. J. McGinn, University of Missouri, MISCON Group Meeting, January 1993.

60. "Aspects of $\mathrm{Y}_{2} \mathrm{BaCuO}_{5}$ Particle Coarsening During Melt Processing of YBa2 $\mathrm{Cu}_{3} \mathrm{O}_{7-\mathrm{x}}$, C. Varanasi and P. J. McGinn. TMS Annual Meeting. Denver, CO, February 1993.

61. "Flux Pinning Associated with $\mathrm{Y}_{2} \mathrm{BaCuO}_{5}$ Precipitates in Melt Processed YBa2 $\mathrm{Cu}_{3} \mathrm{O}_{7-\mathrm{x}}$ ", S. Sengupta, D. Shi, J. S. Lou, C. Varanasi, P. J. McGinn, U. Balachandran, and K. C. Goretta, ICMC Conference, Santa Fe, NM, July 1993.

62. "Microstructural Dependence of Critical Current Density in Melt Textured YBa2Cu3O6+x Superconductors", P. McGinn, MISCON Group Meeting, University of Notre Dame, Notre Dame, IN, July 1993.

63. "Considerations in the Processing of Long Lengths of Melt Textured $\mathrm{YBa}_{2} \mathrm{Cu}_{3} \mathrm{O}_{6+\mathrm{x}}$ Wires", P. J. McGinn, C. Varanasi, and D. Balkin, TMS Fall Meeting, Pittsburgh, PA, November 1993.

64. "Effective Activation Energy in the Bi-Sr-Ca-Cu-O Systems", S. Sengupta, Donglu Shi, Zuning Wang, M. Smith, and P. J. McGinn, IEEE Transactions on Applied Superconductivity, 3, No. 1, 1226$1228,1993$.

65. "Flux Pinning by 211 Precipitates in Melt-processed $\mathrm{YBa}_{2} \mathrm{Cu}_{3} \mathrm{O}_{\mathrm{x}}$ ", Donglu Shi, S. Sengupta, M. Smith, Z. Wang, A. C. Biondo, U. Balachandran, and K. C. Goretta, IEEE Transactions on Applied Superconductivity 3, No. 1, 1034-1036, 1993. (McGinn Group). 
66. "Critical Current Density, Irreversibility Line, and Flux Creep Activation Energy in Silver-Sheathed $\mathrm{Bi}_{2} \mathrm{Sr}_{2} \mathrm{Ca}_{2} \mathrm{Cu}_{3} \mathrm{O}_{x}$ Superconducting Tapes", Donglu Shi, $Z$. Wang, S. Sengupta, M. Smith, L. F. Goodrich, S. X. Dou, H. K. Liu, and Y. C. Guo, IEEE Transactions on Applied Superconductivity 3, No. 1, 1194-1196, 1993. (McGinn Group).

67. "Critical Current of High $T_{c}$ Superconducting Thin Film of YBCO: The Enhancement of Pinning Effect by Silver Particles", C. Zhong, T. B. Ekkens, S. Nolen, and S. Rugglero, 1993 March APS Meeting.

68. "Synthesis and Properties of $\mathrm{YBa}_{2} \mathrm{Cu}_{3} \mathrm{O}_{7}$ with $\mathrm{ZrO}_{2}$ or $\mathrm{MgO}$ by Anionic Oxddation-Reduction", Siyoung Choi and Patrick K. Gallagher, Am. Ceram. Soc. An. Meeting, Cincinnat, OH, April 20, 1993.

69. "Inter-Plane Josephson Coupling of Thermally Excited Vortices in $\mathrm{Bi}_{2} \mathrm{Sr}_{2} \mathrm{CaCu}_{2} \mathrm{O} 8-\delta^{\prime \prime}$, Y. M. Wan, S. E. Hebboul, D. C. Harris, and J. C. Garland, Bull. Am. Phys. Soc. 38, 224, 1993.

70. "Evidence for a Two-Stage Zero-Field Resistive Transition in $\mathrm{Bi}_{2} \mathrm{Sr}_{2} \mathrm{CaCu}_{2} \mathrm{O} 8$ ", S. E. Hebboul, Y. M. Wan, D. C. Harris, and J. C. Garland, Bull. Am. Phys. Soc. 38, 224, 1993.

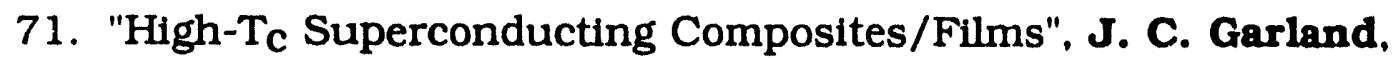
University of Notre Dame, Notre Dame, IN, July 7, 1993.

72. "Interlayer Josephson Coupling of Thermally Excited Vortices in $\mathrm{Bi}_{2} \mathrm{Sr}_{2} \mathrm{CaCu}_{2} \mathrm{O} 8-\delta^{\prime \prime}$, J. C. Garland, University of Michigan, Ann Arbor, October 25, 1993.

73. "Infrared Reflectance of Doped YBCO Films: Where is the Gap?", T. R. Lemberger, Gordon Conference on Superconductivity, January 1993.

74. "Penetration Depth $\lambda(\mathrm{T})$ of $\mathrm{YBa}_{2} \mathrm{Cu} 3 \mathrm{O}_{7-\delta} \delta$ Films Determined from Linear Structures", J. Y. Lee and T. R. Lemberger, Bull. Am. Phys. Soc. 38, 1993.

75. "Infrared Reflectance and Penetration Depth of Doped Oxide Superconductors", T. R. Lemberger, Indiana University, April 1993.

76. "Measurement of Penetration Depth $\lambda(\mathrm{T})$ in $\mathrm{YBa}_{2} \mathrm{Cu}_{3} \mathrm{O}_{7-} \delta$ Thin Film", JuYoung Lee and Thomas R. Lemberger, 20th International Conference on Low-Temperature Physics, August 1993. 
77. "The Midinfrared Conductivity Determined from the Infrared Reflectance of $\mathrm{YBa}_{2}\left(\mathrm{Cu}_{1-\mathrm{xNi}}\right)_{3} \mathrm{O}_{7-\delta}$ Fllms", Jin-Tae Kim, Eric R. Ulm, and Thomas R. Lemberger, 20th International Conference on Low-Temperature Physics, August 1993.

78. "Magnetic Penetration Depth $\lambda(\mathrm{T})$ in Ni-doped $\mathrm{YBa}_{2}\left(\mathrm{Cu}_{1-\mathbf{x}} \mathrm{Ni}_{\mathbf{X}}\right)_{3} \mathrm{O}_{7}$ ", Eric R. Ulm, Jin-Tae Kim, and Thomas R. Lemberger, 20th International Conference on Low-Temperature Physics, August 1993.

79. "Properties of Films of High-Temperature Superconductors", T. R. Lemberger, Ohio State University, October 1993.

80. "Infrared Reflectance and Penetration Depth of Doped Oxide Superconductors", T. R. Lemberger, University of Florida, November 1993.

81. "Stability of Bi-Sr-Ca-Cu-O Thick Films at Elevated Oxygen Pressures", K. H. Sandhage and D. H. Chmielewski, Workshop on Phase Equilibria of High-T $T_{c}$ Superconductors, Santa Fe, NM, June 4, 1993.

82. "The Influence of Elevated Pressure Annealing on the Stability of $\mathrm{Bi}_{2} \mathrm{Sr}_{2} \mathrm{Ca}_{1} \mathrm{Cu}_{2} \mathrm{Oy} "$, D. H. Chmielewski and K. H. Sandhage, TMS/ASM Fall Meeting, Pittsburgh, PA, October 18, 1993.

83. "High Temperature Physical Chemistry Involved in the Processing of Bulk Superconducting Oxdde Wires/Tapes", K. H. Sandhage, IAP Research, Inc., Dayton, OH, November 18, 1993.

84. "Dynamical Properties of Josephson Junction Arrays: I. Shapiro Steps in Josephson Junction Arrays", David Stroud, CTP Workshop on Statistical Physics, Seoul, South Korea, January 13, 1993.

85. "Dynamical Properties of Josephson Junction Arrays: II. Bosc Glass Transition in Josephson Junction Arrays, and Behavior of Underdamped Arrays", Davld Stroud, CTP Workshop on Statistical Physics, Seoul, South Korea, January 14, 1993.

86. "Dynamics of Josephson Networks", David Stroud, General Colloquium, Georgia Institute of Technology, Atlanta, GA, February 24, 1993.

87. "I-V Characteristics and Phase Transitions in Model High Temperature Superconductors with Columnar Defects", K. H. Lee, D. Stroud, and S. M. Girvin, APS March Meeting, Seattle, WA, March 1993. 
88. "Resistive Steps in Josephson Junction Arrays", Wenbin Yu, K. H. Lee, and D. Stroud, APS March Meeting, Seattle, WA., 1993.

89. "I-V Characteristics of Model High-Temperature Superconductors with Columnar Defects", K. H. Lee, D. Stroud, and S. M. Girvin, Bull. Am. Phys. Soc. 38, 801, 1993.

90. "Flux Flow, Flux Pinning, and Phase Transitions", David Stroud, Bull. Am. Phys. Soc. 38, 123, 1993.

91. "Dynamical Properties of Josephson Systems", Davdd Stroud, Condensed Matter Seminar, Purdue University, West Lafayette, IN, April 26, 1993.

92. "Weak-Coupled Superconductors", David Stroud, Condensed Matter Seminar, University of Wisconsin, Madison, WI, November 4, 1993.

93. "Orientation Texture in YBCO Films Synthesized from Amorphous Precursors", Chandra Venkatraman and Gerald L. Liedl, TMS Annual Meeting.

94. "Transport Properties of High $T_{c}$ Superconductors", G. F. Giuliand, Midwest Superconductivity Consortium January Meeting, Columbia, MO, January 1993.

95. "Critical Current in a Lateral Josephson Junction between Layered Superconductors", G. F. Giuliani, APS March Meeting, Seattle, WA, March 1993.

96. "Critical Current of a Josephson Junction in Layered Superconductors", G. F. Giuliani, APS March Meeting, Seattle, WA, March 1993.

97. "Cahotic Vortex Penetration in Layered Superconductors", G. F. Giuliani, International Centre for Theoretical Physics, Scuola Normale Superiore, Pisa, Italy, June 1993.

98. "Antiparallel Spin Correlations in the Landau Theory of Fermi Liquids", G. F. Giuliani, Symposium in Honor of John J. Quinn, Brown University, Providence, Rhode Island, September 1993.

99. "Critical Current of a Josephson Junction and Layered Superconductor with Anisotropic Vortices", M. Fistul, Argonne National Laboratory, Chicago, IL, June 1993. (Gluliani Group)

100. "Interaction of a Long Josephson Junction with Abrikosov Vortices", M. Fistul, APS March Meeting, Seattle, WA, March 1993. (Giuliani Group) 


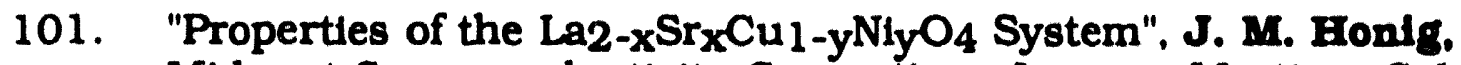
Midwest Superconductivity Consortium January Meeting, Columbia. MO, January 1993.

102. "Real Space Pairing of Heavy Fermion Materials and High $T_{C}$ Superconductors", J. M. Fonig, Midwest Superconductivity Consortium January Meeting, Columbia, MO, January 1993.

103. "Why Solids Undergo the Trauma of Metal Insulator Transitions", J. M. Honig, Superconductor Applications Association, San Jose, CA. February 1-3, 1993.

104. "Effect of Fluortnation on La2-xSr $\mathrm{SrCuO}_{4}$ Superconductors", J. M. Honig. Department of Chemistry, San Jose State University, San Jose, CA, February 4, 1993.

105. "The Trauma of Phase Transitions in Solids", J. M. Honig, Eastern Kentucky Section of the American Chemical Society, Richmond, KY, February 9, 1993.

106. "Single Crystal Growth and Characterization of Electrical Properties in Transition Metal Oxddes". J. M. Honig, Department of Chemistry, Eastern Kentucky University, Richmond, KY, February 10, 1993.

107. "The Trauma of Phase Transitions in Solids", J. M. Honig. Department of Physics, University of Kentucky, Lexington, KY, February 11, 1993.

108. "Electron Correlation Phenomena in Selected Transition Metal Oxides", J. M. Honig, International Conference on Magnetism. Poznán, Poland, June 21-24, 1993.

109. "Electron Correlation Phenomena in Selected Transition Metal Oxides", J. M. Honig, Conference on Electron Correlation Phenomena, Blayistok, Poland. June 28-30, 1993.

110. "Correlation Effects in Electrical Transport Properties in Inorganic Transition Metal Compounds", J. M. Honig, Meeting on Current Topics in Solid State Chemistry, Indian Institute of Science, Bangalore, India, December 14-17, 1993.

111. "Correlation Effects in Electrical Transport Properties in Inorganic Transition Metal Compounds", J. M. Honig, Regional Research Laboratory Thiruvananthapuram, India, December 20, 1993.

112. "Application of Statistical Spin Liquid Concept to HighTemperature Superconductivity", K. Byczuk and J. SpaXek. International Conference on Magnetism, Poznan, Poland, 1993. 
113. "Strongly Correlated Fermions at Low Temperatures", J. 8palek, J. Karbowskd, W. Wojclk, European Physical Society Condensed Matter Division Conference, Regensburg, Germany, March 29 - April 2. 1993.

114. Invited seminar at the University of Illinois at Chicago, Department of Physics, Chicago, IL, J. 8pal el, September 20, 1993.

115. "Texture Development in $\mathrm{XBa}_{2} \mathrm{Cu}_{3} \mathrm{O}_{7-\delta}$ Fllms Synthesized from Metallo-Organic Precursors", Chandra Venkatraman and Gerald L. Led1, 95th ACerS Annual Meeting, Cincinnati, OH, April 18-22, 1993.

116. "Effect of Processing Parameters on Texture Development in YBa2Cu307- $\delta$ Films Synthesized from Metallo-Organic Precursors", Chandra Venkatraman and Gerald L. Lled1, MRS 1993 Fall Meeting. Boston, MA, November 29-December 3, 1993.

117. "Correlation of Magnetic and Transport Behavior in YBaCuO Thin Fulms", M. W. McElfresh, IVth International Conference on

Frontiers in Condensed Matter Physics, Bar-Ilan University, RamatGan, Israel, March 1993.

118. "Magnetic Order in the Frustrated 2D Heisenberg Antiferromagnet: La2-x $\mathrm{Sr}_{\mathbf{x}} \mathrm{NiO}_{4+\delta "}$ ", M. Darwin, M. McElfresh, P. Metcalf, J. Honig. and B. Crooker, APS March Meeting, Seattle, WA, March 1993.

119. "Irreversibility Line in YBaCuO Thin Films: Correlation of Transport and Magnetic Behavior", J. Deak, M. McElfresh, John R. Clem. Zhidong Hao, M. Konczykowskd, R. Muenchausen, S. Foltyn, and R. Dyne, APS March Meeting, Seattle, WA, March 1993.

120. "Reduction of $T_{C}$ at YBCO Bicrystal Thin Film Grain Boundaries", A. Lewicki, Shi Li, M. Darwin, C. Kovach, J. Deak, Lifang Hou, and M. McElfresh, APS March Meeting, Seattle, WA, March 1993.

121. "Effect of Transport Currents on the Critical State in YBCO Thin Films", M. Darwin, J. Deak, L. Hou, M. McElfresh, E. Zeldov, and John R. Clem, APS March Meeting, Seattle, WA, March 1993.

122. "Synthesis and Characterization of $\mathrm{Ni}_{3} \mathrm{~S}_{2}$ Single Crystals", P. Metcalf, P. Fanwick, Z. Kakol, B. C. Crooker, M. McElfresh, N. Otsuka, and J. M. Honig. APS March Meeting, Seattle, WA, March 1993. 
123. "Irreversibillty Line in High Temperature Superconductors", M. W. McElfresh, Materials Science Seminar, University of Wisconsin, Madison, WI, April 22, 1993.

124. "Effect of Transport Currents on the Critical State in YBCO Thin Fllms", M. W. McElfreeh, CISE Laboratories, Milan, Italy, June 1993.

125. "Effect of Transport Currents on the Critical State in YBCO Thin Fulms", M. W. McElfresh, Orsay University, Orsay, France, June 1993.

126. "Magnetic Properties of Superconductors: The Basics", Workshop on "Type II Superconductors: Basic Properties, Measurements, and Selected Advanced Topics", M. W. McElfresh. University of Notre Dame, Notre Dame. IN. July 8-9, 1993.

127. "Properties of the Irreversibility Line: An Update", M. W. McElfresh, Invited Presentation at Los Alamos National Laboratory, Los Alamos, NM, August 11, 1993.

128. "Properties of the Irreversibillity Line: An Update", M. W. McElfresh, Invited Presentation at Sandia National Laboratory. Albuquerque, NM, August 12, 1993.

129. "Formation of Tetragonal $\mathrm{YBa}_{2} \mathrm{Cu}_{3} \mathrm{O}_{7-\delta} \delta$ from an Undercooled Melt", J. R. Olive, W. H. Hofmeister, R. J. Bayuzick, G. Carro, J. P. McHugh, R. H. Hopkins, M. Vlasse, R. Weber, P. Nordine, and M. McElfresh, MRS 1993 Fall Meeting, Boston, MA.

130. "Vortices in Anisotropic Superconductors", G. Preosti, Argonne National Laboratory, November 1993. (Muzikar Group)

131. "Texture and Microstructure in Ceramics", K. J. Bowman, Riso National Laboratory, Denmark, June 24, 1993.

132. "Texture in Ceramic Materials", K. J. Bowman, Proceedings of the Tenth International Conference on Texture of Materials, Clausthal, Germany, Sept. 1993.

133. "Texture and Microstructure in Ceramic Materials", K. J. Bowman, Case Western, Cleveland, OH, Sept. 14, 1993.

134. "Preferred Orientation of BSCCO Via Centrifugal Slip Casting", G. Steinlage, K. P. Trumble, K. J. Bowman, S. Li, and M. McElfresh, TMS Meeting, Indianapolis, IN, January 11, 1993. 
135. "Structural Stabllity of $\mathrm{Bi}_{2} \mathrm{Sr}_{2} \mathrm{Ca}_{n-1} \mathrm{O}_{2 n+4}$ Compounds: A Study on the Effect of Cation Doping and Oxygen Non-Stolchiometry", $\mathrm{H}$. Zhang and H. Bato, MRS Fall Meeting, Boston, MA, Dec. 1, 1993. 


\section{MISCON PUBLICATIONS \\ January 1993 - December 1993}

(In Print)

1. "I-V Characteristics of High-Temperature Superconductors with Columnar Defects", Mats Wallin and S. M. Girvin, Phys. Rev. B 47, 14642-14645, 1993.

2. "The Universal Conductivity at the Zero-temperature Superconductor-insulator Transition in Two Dimensions: Monte Carlo Calculation", Min-Chul Cha and S. M. Girvin, in Proc. 2nd CTP Workshop on Statistical Physics: KT Transition and Superconducting Arrays, ed. by D. Kim, J.S. Chung, and M.Y. Choi (Min Eum Sa Co., Seoul, 1993), p. 225-239.

3. "Quantum Mechanics and Superconductivity in a Magnetic Field", A. H. MacDonald, Hiroshi Akera, and M. R. Norman, Aust. J. Phys. 46. 333-344, 1993.

4. "Two-Dimensional Vortex Lattice Melting", Jun $\mathrm{Hu}$ and A. H. MacDonald, Phys. Rev. Lett. 71, 432-435, 1993.

5. "Theory of Microwave Transmission and Reflection in Type-II Superconductors in the Mixed State", Mark W. Coffey and John R. Clem, Phys. Rev. B 48, 342-350, 1993.

6. Comment on "From Isotropic to Anisotropic Superconductors: A Scaling Approach", Zhidong Hao and John R. Clem, Phys. Rev. Lett. 71, 301-302, 1993.

7. "Vortex Fluctuations in Polycrystalline $\mathrm{Bi}_{1} .75 \mathrm{~Pb} 0.25 \mathrm{Sr}_{2} \mathrm{Ca}_{2} \mathrm{Cu}_{3} \mathrm{O}_{10}$ ", J. H. Cho, D. C. Johnston, M. Ledvij, and V. G. Kogan, Physica C 212. 419-423, 1993.

8. "Magnetic Field of Vortices Crossing a Superconductor Surface", v. G. Kogan, A. Yu. Simonov, and M. Ledvj, Phys. Rev. B 48, 392-397, 1993.

9. "Role of Vortex Fluctuations in Determining Superconducting Parameters from Magnetization Data for Layered Superconductors", V. G. Kogan, M. Ledvij, A. Yu. Simonov, J. H. Cho, and D. C. Johnston, Phys. Rev. Lett. 70, 1870-1873, 1993. 
10. "Low Temperature Fluctuations of Vortices in Layered

Superconductors", Y. -Q. Song, W. P. Halperin, L. Tonge, T. J.

Marks, M. Ledvij, V. G. Kogan, and L. N. Bulaevskii, Phys. Rev. Lett. 70, 3127-3130, 1993.

11. "Distorted Vortex in Josephson-Coupled Layered Superconductors", L. N. Bulaevskii, M. Ledvij, and V. G. Kogan, Phys. Rev. B 46, 11807-1 1812, 1992.

12. " Observation of a Novel Vortex Structure Driven by Magnetic Interactions Near a Sawtooth Twin Boundary in YBa2Cu3O7- $\delta$ ", P. L. Gammel, C. A. Durán, D. J. Bishop, V. G. Kogan, M. Ledvij, A. Yu. Simonov, J. P. Rice, and D: M. Ginsberg, Phys. Rev. Lett. 69, 3808$3811,1992$.

13. "Layered High-T $\mathrm{T}_{\mathrm{c}}$ Superconductors in an AC Field", A. Yu. Simonov, Physica C 211, 455-466, 1993. (Clem, Kogan Group)

14. "Transport, Structural and Magnetic Properties of the Single-copperoxygen Layer Bi2Sr2-xLaxCuOy System", A. E. Schlögl, J. J. Neumeier, J. Diederichs, C. Allgeier, J. S. Schilling, and W. Yelon, Physica C 216, 417-431, 1993.

15. "Structural and Magnetic Studies on PrBa2Cu2NbO8-y", S. K. Malik, H. Jhans, w. B. Yelon, and J. J. Rhyne, Solid State Communications 85, 849-852, 1993.

16. ""Defect Size Dependence of Critical Current Density Enhancement for Irradiated YBa2Cu3O7- $\delta "$, J. Bechtold, Y. Y. Xue, Z. J. Huang, E. V. Hungerford, P. H. Hor, C. W. Chu, X. K. Maruyama, H. Backe, F. R. Buskirk, S. M. Connors, D. D. Snyder, Y. C. Jean, and J. W. Farmer, Physica C 191, 199-204, 1992.

17. *"Flux Pinning in $\mathrm{YBa}_{2} \mathrm{Cu}_{3} \mathrm{O}_{7-\delta} \delta$ Single Crystals: Neutron Irradiation and Annealing", B. M. Vlcek, M. C. Frischherz, S. Fleshler, U. Welp, J. Z. Liu, J. Downey, K. G. Vandervoort, G. W. Crabtree, M. A. Kirk, J. Giapintzakis, and J. Farmer, Phys. Rev. B 46, 6441-6450, 1992.

18. "Vortex Kink Motion Versus Phase Slip and Anisotropic Flux Flow in Superconducting $\mathrm{YBa}_{2} \mathrm{Cu}_{3} \mathrm{O}_{7}-\delta "$, H. A. Blackstead, Supercond. Sci. Technol. 6, 579-583, 1993.

*Published in 1992, Reported in 1993 
19. "Field Induced Resistivity in Crystalline $\mathrm{YBa}_{2} \mathrm{Cu}_{3} \mathrm{O} 7-\delta$ : Evidence for Nano-Scale Junction Structures", H. A. Blackstead, D. G. Keiffer, M. D. Lan, and J. Z. Liu, Superlattices and Microstructures, 13, 3, 279-284, 1993.

20. "Anisotropic Resistivity in the Mixed State of $\mathrm{YBa}_{2} \mathrm{Cu}_{3} \mathrm{O} 7-\delta$ ", H. A. Blackstead, Physica C 209, 437-441, 1993.

21. "Current and Angular-Dependent Transport in the Mixed State of $\mathrm{Bi}_{2} \mathrm{Sr}_{2} \mathrm{CaCu}_{2} \mathrm{O} 8+\delta "$, H. A. Blackstead, Solid State Communications 87, 35-39, 1993.

22. "Anisotropic Resistivity in the Mixed State of Crystalline $\mathrm{YBa}_{2} \mathrm{Cu}_{3} \mathrm{O}_{7+\delta} \delta$, H. A. Blackstead, Phys. Rev. B 47, 11411-11415, 1993.

23. "Observation of Giant Flux-Flow in Superconducting $\mathrm{YBa}_{2} \mathrm{Cu}_{3} \mathrm{O}_{7-\delta}$ Films", H. A Blackstead, D. B. Pulling, J. S. Horwitz, and D. B. Chrisey, Solid State Communications 88, 671-675, 1993.

24. "Electron Spin Resonance in Superconducting Melt-Textured GdBa2Cu307- $\delta$ : Evidence Against Uniform Flux Entangling", H. A. Blackstead, D. B. Pulling, and C. A. Clough, Phys. Rev. B 47, 89788985, 1993.

25. "Crystal Structure, Microstructure, Surface Morphology and Transport Properties of $\mathrm{Er}_{5} \mathrm{Ba}_{7} \mathrm{Cu} 12 \mathrm{O}$ y High-Temperature Superconductor Thin Films", K. M. Choudhary, P. Seshadri, J. Bae, M. Black, and A. Lewicki, Phys. Rev. B 48, 9697-9709, 1993.

26. "Nonlinear $\mathrm{U}(\mathrm{j})$ Law from Magnetic Relaxation in $\mathrm{Bi}_{2} \mathrm{Sr}_{2} \mathrm{CaCu}_{2} \mathrm{O}_{\mathbf{x}}$ Single Crystals: Flux Motion Through Double-kink Formation of the Pancake Vortices", S. Sengupta, Donglu Shi, Sergei Sergeenkov, and P. J. McGinn, Phys. Rev. B 48, 9, 6736-6739, 1993.

27. "Extremely Fine Precipitates and Flux Pinning in Melt-Processed YBa2Cu3Ox", Donglu Shi, S. Sengupta, J. S. Luo, C. Varanasi, and P. J. McGinn, Physica C 213, 179-184, 1993.

28. "Effect of $\mathrm{YBa}_{2} \mathrm{Cu}_{3} \mathrm{O}_{7-\mathrm{x}}$ Grain Size on the Nucleation of $\mathrm{Y}_{2} \mathrm{BaCuO}_{5}$ During Melt Texturing", C. Varanasi and P. J. McGinn, Materials Letters 17, 205-209, 1993. 
29. "Flux Pinning by 211 Precipitates in Melt-Processed $\mathrm{YBa}_{2} \mathrm{Cu}_{3} \mathrm{O}_{\mathrm{x}}$ ", Donglu Shi, S. Sengupta, M. Smith, Z, Wang, A. C. Biondo, U. Balachandran, and K. C. Goretta, (McGinn Group), Applied Superconductivity 3,1034-1036, 1993.

30. "Effective Activation Energy in the Bi-Sr-Ca-Cu-O Systems", S. Sengupta, Donglu Shi, Zuning Wang, M. Smith, and P. J. McGinn, Applied Superconductivity 3, 1226-1228, 1993.

31. "The Effect of $\mathrm{PtO}_{2} \cdot \mathrm{H}_{2} \mathrm{O}$ Additions on the $\mathrm{Y}_{2} \mathrm{BaCuO}_{5}$ Morphology in Melt Textured YBa2Cu3O7-x", C. Varanasi and P. J. McGinn, Physica C 207, 79-84, 1993.

32. "Effective Flux-Creep Activation Energy and Anisotropy in a $\mathrm{Bi}_{2} \mathrm{Sr}_{2} \mathrm{CaCu}_{2} \mathrm{O}_{x}$ Single Crystal", M. E. Smith, Donglu Shi, S. Sengupta, and Z. Wang (McGinn Group), Applied Superconductivity 1,1/2, 151-156, 1993.

33. "Magnetic Relaxation and Intrinsic Pinning in a Single Crystal of $\mathrm{Bi}_{2} \mathrm{Sr}_{2} \mathrm{CaCu}_{2} \mathrm{O}_{\mathrm{x}}$ ", S. Sengupta, Donglu Shi, Zuning Wang, M. E. Smith, S. Salem-Sugui, Jr., and P. J. McGinn, Phys. Rev. B 47, 5414-5418, 1993.

34. "U-j Relationship in Type-II Superconductors", S. Sengupta, Donglu Shi, Zuning Wang, M. E. Srnith, and P. J. McGinn, Phys. Rev. B 47. 5165-5169, 1993.

35. *Flux Pinning in Hot Isostatically Pressed $\mathrm{Bi}_{2} \mathrm{Sr}_{2} \mathrm{CaCu}_{2} \mathrm{O}$ ", D. J. Miller, S. Sengupta, J. D. Hettinger, D. Shi, K. E. Gray, A. S. Nash, and K. C. Goretta (McGinn Group), Appl. Phys. Lett. 61, 23, 28232825, 1992.

36. "Interlayer Josephson Coupling of Thermally Excited Vortices in Bi2Sr2CaCu2O8-y", Y. M. Wan, S. E. Hebboul, D. C. Harris, and J. C. Garland, Phys. Rev. Lett. 71, 157-160, 1993.

37. "rf Power Dependence of Subharmonic Voltage Spectra of TwoDimensional Josephson-Junction Arrays", S. E. Hebboul and J. C. Garland, Phys. Rev. B 47, 5190-5195, 1993.

38. *"Evidence for Scaling Invariance and Universality of the Irreversibility Line of High Temperature Superconductors", C. C. Almasan, M. C. de Andrade, Y. Dalichaouch, J. J. Neumeier, C. L. Seaman, M. B. Maple, R. P. Guertin, M. V. Kuric, and J. C. Garland, Phys. Rev. Lett. 69, 3812-3815, 1992.

*Published in 1992, Reported in 1993 
39. "Penetration Depth $\lambda(\mathrm{T})$ of $\mathrm{YBa}_{2} \mathrm{Cu}_{3} \mathrm{O}_{7-\delta} \delta$ Films Determined from the Kinetic Inductance", JuYoung Lee and Thomas R. Lemberger, Appl. Phys. Lett. 62, 2419-2421, 1993.

40. "Vortex Correlations in the Superconducting Transition of $\mathrm{YBa}_{2}\left(\mathrm{Cu}_{1-}\right.$ $\left.\mathrm{xAl}_{\mathbf{X}}\right)_{307-\delta}$ Films in High Magnetic Fields", Dimitrios G. Xenikos, Jin-Tae Kim, and Thomas R. Lemberger, Phys. Rev. B 48, 77427745, 1993.

41. "Absence of a Gap in the Infrared Reflectance of Ni-doped YBa2 $\mathrm{Cu}_{3} \mathrm{O}_{7-\delta} \delta$ Films", Michael J. Sumner, Jin-Tae Kim, and Thomas R. Lemberger, Phys. Rev. B 47, 12248-12256, 1993.

42. "Vortex Motion in Josephson-junction Arrays near $f=0$ and $f=1 / 2$ ", Wenbin Yu, K. H. Lee, and D. Stroud, Phys. Rev. B 47, 5906-5914, 1993.

43. "Flux Pinning and Phase Transitions in Model High-Temperature Superconductors with Columnar Defects", K. H. Lee, D. Stroud, and S. M. Girvin, Phy. Rev. B 48, 1233-1243, 1993.

44. "Flux-lattice Melting in Layered Superconductors", R. Šášik and D. Stroud, Phys. Rev. B 48, 9938-9941, 1993.

45. "Dynamics of Josephson Networks", D. Stroud, K. H. Lee, and Wenbin Yu, in KT Transition and Josephson Junction Arrays, edited by D. Kim, J. S. Chung, and M. Y. Choi (Min Eum Sa, Seoul), pp. 95-152, 1993.

46. "Critical Current of a Josephson Junction in Layered Superconductors", Mikhail V. Fistul and Gabriele F. Giuliani, Phys. Rev. B 47, 11341-11345, 1993.

47. *"Critical Current of a Josephson Junction with Spatial Fluctuations of the Order Parameter", V. V. Dorin and M. V. Fistul, Phys. Rev. B. 46, 13951-13955, 1992. (Giuliani Group)

48. "Synthesis and Characterization of $\mathrm{Ni}_{3} \mathrm{~S}_{2}$ Single Crystals", P. A. Metcalf, P. Fanwick, Z. Kakol, and J. M. Honig. Journal of Solid State Chemistry 104, 81-87, 1993.

*Published in 1992, Reported in 1993 
49. *"Fluorination of $\mathrm{La2}_{-\mathrm{x}} \mathrm{Ca}_{\mathrm{x}} \mathrm{CuO}_{4+\delta}$ : Exhibition of Superconductivity for $\chi \leq 0.05$ and Enhancement of $T_{c}$ for $\chi \geq 0.1 "$. V. Bhat and J. $\mathbf{M}$. Honig, Indian Journal of Pure \& Applied Physics 30, 498-501, 1992.

50. "Incommensurate Spin Density Wave in Metallic V2-yO3", Wei Bao, C. Broholm, S. A. Carter, T. F. Rosenbaum, G. Aeppli, S. F. Trevino, P. Metcalf, J. M. Honig, and J. Spal ek, Phys. Rev. Lett. 71, 766-769, 1993.

51. "Doping Dependence of the a-b-plane Optical Conductivity of Single Crystal La2-x $\mathrm{Sr}_{\mathrm{X}} \mathrm{NiO} 4+\delta "$, Xiang-Xin Bi, P. C. Eklund, and J. M. Honig, Phys. Rev. B 48, 3470-3478, 1993.

52. "Oriented Growth in Oxide Thin Film Heterostructures", V. R. Iyer, E. P, Kvam, D. L. Perry, R. Ramesh, T. D. Sands, and V. Keramidas, Scripta Metallurgica 29, 885-888, 1993.

53. "Crystalline Linkage and Defect Structures in Bulk Zone Melt Textured $\mathrm{YBa}_{2} \mathrm{Cu}_{3} \mathrm{O}_{7}$ Observed by Transmission Electron Microscopy", A. Zanota, E. P. Kvam, D. Balkin, and P. J. McGinn, Appl. Phys. Lett. 62, 2722-2724, 1993.

54. "Identifying the Loss of Critical Current Density in $\mathrm{YBa}_{2} \mathrm{Cu}_{3} \mathrm{O}_{7}$ Thin Films", J. Deak, M. McElfresh, John R. Clem, Zhidong Hao, M. Konczykowski, R. Muenchausen, S. Foltyn, and R. Dye, Phys. Rev. B 47, 8377-8380, 1993.

55. "Density-of-States Fine Structure in the Tunneling Conductance of Y-Ba-Cu-O Films: A Comparison Between Experiment and Theory", T. G. Miller, M. McElfresh, and R. Reifenberger, Phys. Rev. B 48, 7499-7504, 1993.

56. "Effect of Flux Creep on the Temperature Dependence of the Current Density in Y-Ba-Cu-O Crystals", J. R. Thompson, Yang Ren Sun, L. Civale, A. P. Malozemoff, M. W. McElfresh, A. D. Marwick, and F. Holtzberg, Phys. Rev. B 47, 14440-14447, 1993.

57. "Frequency Independence of the Vortex-glass Transition in YBa2Cu3O7- $\delta$ Thin Films", J. Deak, M. McElfresh, R. Muenchausen, S. Foltyn, and R. Dye, Phys. Rev. B 48, 1337-1340, 1993.

${ }^{*}$ Published in 1992, Reported in 1993 
58. "Structure and Properties of the LaCuO3- $\delta$ Perovskites", J. F. Bringley, B. A. Scott, S. J. La Placa, T. R. McGuire, F. Mehran, M. W. McElfresh, and D. E. Cox, Phys. Rev. B 47, 15269-15275, 1993.

59. "Pressure Dependence of the Static Magnetic Susceptibility of the Heavy-Fermion Superconductor UBe 13", M. McElfresh, M. B. Maple, J. O. Willis, D. Schiferl, J. L. Smith, Z. FIsk, and D. L. Cox, Phys. Rev. B 48, 10395-10403, 1993.

60. "Effect of Transport Currents on the Critical State of YBa2 $\mathrm{Cu}_{3} \mathrm{O} 7-\delta$ Thin Films", M. Darwin, J. Deak, L. Hou, M. McElfresh, E. Zeldov, John R. Clem, and M. Indenbom, Phys. Rev. B 48, 13192-13195, 1993.

61. "Enhancement of $\mathrm{T}_{\mathrm{C}}$ in $\mathrm{YBa}_{2} \mathrm{Cu}_{3} \mathrm{O}_{7}$ by Chemical Substitution in Bulk and Thin Film Materials: Implications for the Upper Critical Magnetic Field", J. J. Neumeier, M. W. McElfresh, Lifang Hou, and A. Lewicki, Applied Superconductivity 1, 1279-1288, 1993.

62. "Minimum Energy Vortex Configurations in Anisotropic Superconductors", G. Preosti and Paul Muzikar, Phys. Rev. B 48. 9921-9924, 1993.

63. "Structural Stability of Bi-Based Cuprates", Huanbo Zhang and Hiroshi Sato, Physica C 214, 265-271, 1993. 


\section{(Accopted for Publication)}

1. "Anisotropic Superconductors: Fundamentals of Vortices in Layered Superconductors", John R. Clem, to appear in the Proceedings of the NATO Advanced Study Institute.

2. "X-ray Diffraction Studies of Epitaxy in Orthorhombic-

$\mathrm{DyMnO}_{3} / \mathrm{Er}_{1} \mathrm{Ba}_{2} \mathrm{Cu}_{3} \mathrm{O}_{7-\delta} \mathrm{Thin}$ Fulm Heterostructures on LaAlO3(100) Substrates", P. Seshadri and K. M. Choudhary, to appear in Physica C.

3. "Growth Morphologies of (001), (100) and (010) Oriented Er5Ba7Cu $12 \mathrm{O}_{y}$ High-Temperature Superconductor Thin Films on Various Substrates", K. M. Choudhary, P. Seshadri, and M. Black, to appear in J. Vacuum Sci. and Technology B.

4. "Effects of the C-axis Resistive Transition Width on the Secondary Voltage of $\mathrm{Bi}_{2} \mathrm{Sr}_{2} \mathrm{CaCu}_{2} \mathrm{O} 8-\mathrm{y} "$, Y. M. Wan. S. E. Hebboul, D. C. Harris, and J. C. Garland, to appear in Physica B.

5. "DC Magnetization of Random $\mathrm{Y}_{1} \mathrm{Ba}_{2} \mathrm{Cu}_{3} \mathrm{O} 8-\delta / \mathrm{Ag}$ Bulk Composites", C. L. Ash, D. C. Harris, J. J. Calabrese, and J. C. Garland, to appear in Physica B.

6. "Fabrication and Measurements of High $T_{c}$ Weak Links", S. T. Herbert, S. E. Hebboul, and J. C. Garland, to appear in Physica B.

7. "Irreversibility Line in YBaCuO Thin Films: Correlation of Transport and Magnetic Behavior", J. Deak, M. McElfresh, John R. Clem, Zhidong Hao, M. Konczykowski, R. Muenchausen, S. Foltyn, and R. Dye, accepted by Phys. Rev. B.

8. "The Penetration Depth and Impurity Scattering in Unconventional Superconductors: $\mathrm{T}=0$ Results", Heesang Kim. Gianfranco Preosti, and Paul Muzikar, accepted.

9. "Preferred Orientation of BSCCO via Centrifugal Slip Casting", Greg Steinlage, Ryan Roeder, Kevin Trumble, Keith Bowman, Shi Li, and Mike McElfresh, accepted by J. Materials Research. 
1. "Synthesis, Structural Characterization. Thermolysis and Volatility Study of Tricarbomethoxymethanate Complexes of Copper (II) and Barium(II)", David A. Barter, Kenneth G. Caulton, Wen-Chung Chiang, Malcolm E. Chisholm, Vincent F. DiStasi, Sylvain G. Dutremez, James D. Martin, and William E. Streib, submitted.

2. "Synthesis and Structural Characterization of $\mathrm{Cu}\left[\mathrm{CH}_{3} \mathrm{C}(\mathrm{O}) \mathrm{CHC}-\right.$ $\left(\mathrm{NCH}_{2} \mathrm{CH}_{2} \mathrm{OCH}_{3}\right)_{\mathrm{CH}} \mathrm{CH}_{2}$, a Volatile $\beta$-Ketoiminate Complex Sultable for Copper Metal MOCVD", David A. Baxter, Kenneth G. Caulton, Wen-Chung Chiang, Malcolm H. Chisholm, Vincent F. DiStasi, Sylvain G. Dutremez, and Kirsten Folting, submitted.

3. "Correlations in Two-Dimensional Vortex Liquids", Jun Hu and A. H. MacDonald, submitted.

4. "Bound Electron Pairs in Two Dimensions at Quantizing Magnetic Fields", H. Akera, A. H. MacDonald, and D. Yoshioka, submitted.

5. "The Superconductor-insulator Transition in 2D Dirty Boson Systems", Mats Wallin, Erik S. Sфrensen, S. M. Girvin, and A. P. Young, submitted.

6. "Universal Conductivity in the Boson Hubbard Model in a Magnetic Fleld", Min-Chul Cha and S. M. Girvin, submitted.

7. "Magnetization and Transport Currents in Thin Superconducting Films", E. Zeldov, John R. Clem, M. McElfresh, and M. Darwin, submitted.

8. "Hysteretic ac Losses and Susceptibility of Thin Superconducting Disks", John R. Clem and Alvaro Sanchez, submitted.

9. "Effects of Anisotropy and Layering in the High-Temperature Superconductors", John R. Clem, submitted to Physica A.

10. "Structural and Magnetic Properties of Intermediate Oxygen Stoichiometry PrBa2Cu3O ${ }_{x}$, S. K. Malik, W. B. Yelon, J. J. Rhyne, W. J. James, Ram Prasad, K. Adhikary, and N. C. Soni, submitted.

11. "Hole Filling, Charge Transfer and Superconductivity in $\mathrm{Nd}_{1+x} \mathrm{Ba}_{2}-$ $x \mathrm{Cu}_{3} \mathrm{O}_{7+\delta "}$. M. J. Kramer, S. I. Yoo, R. W. McCallum, W. B. Yelon, H. Xie, and P. Allenspach, submitted to Physica C. 
12. "Catastrophic Quenching of Superconductivity in Melt-Textured YBa2Cu3O7- $\delta$ with Second Phase Additions", H. A. Blackstead, D. B. Pulling, and P. J. McGinn, submitted to Applied Physics Letters.

13. "Spin Glass Prepared States and the Resistive Transitions of HighTemperature Superconductors", H. A. Blackstead and J. C. Phillips, submitted.

14. "High Field Temperature Dependent Transport in Polycrystalline TI-Ba-Ca-Cu-O", H. A. Blackstead and G. A. Kapustin, submitted.

15. "Flux-Flow and Phase-Slip Resistivity in Two Phase Polycrystalline $\mathrm{B}_{1}$ 1.6Pbo.4Sr1.7Ca2 $\mathrm{Cu}_{3} \mathrm{O}_{\mathrm{x}}$ ", H. A. Blackstead, D. B. Pulling, and H. Sato, submitted.

16. "Synthesis of $\mathrm{DyMnO}_{3}$ Thin Films on LaAlO $3(100)$ Substrates by Molecular Beam Epitaxy and Characterization of Their Growth Orientation", K. M. Choudhary, P. Seshadri, C. Venkatraman, submitted to Journal of Crystal Growth.

17. "Synthesis of $\mathrm{DyMnO}_{3}$ Thin Films on $\mathrm{LaAlO}_{3}(100)$ Substrates by Molecular Beam Epitaxy and Characterization of Their Growth Orientation", K. M. Choudhary, P. Seshadri, C. Venkatraman, submitted to Phys. Rev. B.

18. "Crystal Structure of $\mathrm{Er}_{5} \mathrm{Ba}_{7} \mathrm{Cu}_{12} \mathrm{O}_{\mathrm{y}}$ Thin Film High-Temperature Superconductor", K. M. Choudhary, P. Seshadri, and J. Bae, submitted to Physical Review Letters.

19. "Critical Current Density and Microstructure of Melt-Processed $\mathrm{YBa}_{2} \mathrm{Cu}_{3} \mathrm{O}_{\mathrm{X}}$ with $\mathrm{PtO}_{2}$ Additions", C. Varanasi, P. J. McGinn, V. Pavate, and E. P. Kvam, submitted to Physica C.

20. "Considerations in the Processing of Long Lengths of Melt Textured YBa2Cu3O6+x Wires", P. J. McGinn, C. Varanasi, D. Balkin, submitted to Proceedings of the TMS Symposium entitled Processing of Long Lengths of Superconductors, ed. by U. Balachandran, A. Goyal, and E.W. Collings, (TMS, 1994).

21. "The Effect of Er 211 Additions on the Microstructure and Magnetic Properties of Zone Melt Textured Y123", David K. Balkin and Paul J. McGinn, submitted to Superconductor Science and Technology.

22. "An Alternate Method to Introduce Fine $\mathrm{Y}_{2} \mathrm{BaCuO}_{5}$ Precipitates into $\mathrm{YBa}_{2} \mathrm{Cu}_{3} \mathrm{O}_{\mathbf{x}}$ With Enhanced Flux Pinning", C. Varanasi, S. Sengupta, and P. J. McGinn, submitted to Applied Superconductivity. 
23. "Y2BaCuO5 Particle Coarsening During Melt Processing of YBa2Cu3O7-x", C. Varanasi and P. J. McGinn, submitted to Journal of Electrical Materials.

24. "Flux Pinning Associated with $\mathrm{Y}_{2} \mathrm{BaCuO}_{5}$ Precipitates in MeltProcessed YBa2Cu3Ox", S. Sengupta, Donglu Shi, J. S. Luo, V. R. Todt, Z. Wang, C. Varanasi, P. J. McGinn, U. Balachandran, and K. C. Goretta, submitted

25. "A Comparison of $\mathrm{PtO}_{2}$ and $\mathrm{BaSnO}_{3}$ Additions on the Refinement of $\mathrm{Y}_{2} \mathrm{BaCuO}_{5}$ and Magnetization of Textured $\mathrm{YBa}_{2} \mathrm{Cu}_{3} \mathrm{O}_{6+\mathrm{x}}$. $\mathrm{C}$.

Varanasi, M. A. Black, and P. J. McGinn, submitted to Superconductor Science \& Technology.

26. "Effects of Oxygen Partial Pressure on the Crystallization of Amorphous $\mathrm{Bi}-\mathrm{Sr}-\mathrm{Ca}-\mathrm{Cu}-\mathrm{O}$ and $\mathrm{Bi}-\mathrm{Sr}-\mathrm{Ca}-\mathrm{Cu}-\mathrm{O}+\mathrm{Ag}$ ", M. J. Kramer, L. Margulies, S. R. Arrasmith. K. W. Dennis, J. C. Lang, P. K. Gallagher, and R. W. McCallum, submitted.

27. "An Atomic Beam Study of a Superconductor's Magnetic Vortex Lattice". Samuel Dapore-Schwartz, Nathan G. Woodard, and Gregory P. Lafyatis, submitted to Phys. Rev. Lett.

28. "Photon Counting Using a Large Area Avalanche Photodiode Cooled to 100K", Nathan G. Woodard, Eric G. Hufstedler, and Gregory P. Lafyatis, submitted.

29. "Crossover in Temperature Dependence of Penetration Depth $\lambda(T)$ in YBa2Cu $3 \mathrm{O}_{7-\delta} \delta$ Films", JuYoung Lee, Kathleen Paget, and Thomas $\mathbf{R}$. Lemberger, submitted to Phys. Rev. Lett.

30. "Infrared Reflectance of $\mathrm{YBa}_{2}\left(\mathrm{Cu}_{1-\mathrm{xNix}}\right)_{3} \mathrm{O}_{7-\delta}$ Fulms", J.-T. Kim, M. J. Sumner, E. R. Ulm, and T. R. Lemberger, submitted.

31. "Magnetic Penetration Depth in Doped $\mathrm{YBa}_{2}\left(\mathrm{Cu}_{1-\mathrm{x}} \mathrm{M}_{\mathrm{X}}\right)_{3} \mathrm{O}_{7}$ Films", Eric R. Ulm, Jin-Tae Kim, Thomas R. Lemberger, Steve R. Foltyn, and Xindi Wu, submitted.

32. "Vortex Correlations in the Superconducting Transition of $\mathrm{YBa}_{2}\left(\mathrm{Cu}_{1}\right.$ $\left.\mathrm{xAl}_{\mathrm{x}}\right)_{30} \mathrm{O}_{-\delta} \delta$ Films in High Magnetic Fields", Dimitrios G. Xenikos, Jin-Tae Kim, and Thomas R. Lemberger, submitted to Phys. Rev. Lett. 
33. "Thermal Stabllity of T1-Ba-Ca-Cu-O High- $\mathrm{T}_{\mathbf{c}}$ Superconductors", Ken E. Eandhage and Patrick K. Gallngher, to appear in Thallium-Based High-Temperature Superconductors, editors Allen M. Hermann and J. V. Yakhmi.

34. "Flux Lattice Melting, Flux Pinning, and Superfluid Density Tensor of YBa2 $\mathrm{Cu}_{3} \mathrm{O}_{7-\delta ",}$ R. Šásilk and D. 8troud, submitted to Phys. Rev. Lett.

35. "Magnetic Fleld Dependence of the Critical Current of a Layered Superconductor", Mikhall V. Fistul and Gabriele F. Giuliani, submitted.

36. "Two-impurity Anderson Model: Results of a Perturbative Expansion in U", Giuseppe E. Santoro and Gabriele F. Giullani, submitted.

37. "Critical Current of a Josephson Junction in Layered Superconductors", Mikhail V. Fistul and Gabriele F. Giulland, submitted.

38. "Two-impurity Anderson Model: Some Exact Results Within Fermi Lquid Theory", Giuseppe E. Santoro and Gabriele F. Giuliani, submitted.

39. "Critical Current of a Lateral Josephson Junction for Layered Superconductors", Mikhail V. Fistul and Gabriele F. Giuliani, submitted.

40. "Reclassification of Early Transition Metal Oxides: Absence of True Mott-Hubbard Materials", J.-H. Park, L. H. TJeng, J. W. Allen, R. Claessen, C. T. Chen, P. Metcalf, and H. R. Harrison, submitted. (Honig Group)

41. "Paramagnetism, Antiferromagnetism and Superconductivity in $\mathrm{La}_{2} \mathrm{NiO}_{4}$ ", J. V. Acrivos, M. Chen Lei, C. Jiang, H. Nguyen, P. Metcalf, and J. M. Honig, submitted.

42. "Melting Curve of Condensed ${ }^{3} \mathrm{He}$ as a Mott-Hubbard Localization Boundary for He Atoms: Elementary Analysis and Comparison with Experiment", J. Spalek, submitted.

43. "Application of Statistical Spin Liquid Concept to High-Temperature Superconductivity", K. Byczuk and J. Spalek, submitted.

44. "Interorbital Pairing for Heavy Fermions and Universal Scaling of Their Basic Characteristics", J. Karbowski and J. Spayek, submitted. 
45. "High-Temperature Superconductor as a Paired State of Statistical Spin Liquid", K. Byczuk and J. Spal ek, submitted to Phys. Rev. B.

46. "Strongly Correlated Fermions at Low Temperatures", J. Spalek, K. Byczuk, J. Karbowski, and W. Wójcik, submitted to Physica Scripta.

47. "Dislocation-Induced Flux Pinning Through Strain Interactions in $\mathrm{YBa}_{2} \mathrm{Cu}_{3} \mathrm{O}_{7}$, E. P. Kram and V. Pavate, submitted to Physica C.

48. "Texture Development in $\mathrm{YBa}_{2} \mathrm{Cu}_{3} \mathrm{O}_{7-\delta}$ Fulms Synthesized from Metallo-organic Precursors", C. Venkatraman, submitted. (Lledi, Bement Group)

49. "Synthesis of YBa2Cu3O7- $\delta$ Films from Metallo-Organic Precursors", C. Venkatraman and G. L. Lledi, submitted to J. Am. Ceram. Soc.

50. "Mechanism of Development and Control of Orientation Texture in $\mathrm{YBa}_{2} \mathrm{Cu}_{3} \mathrm{O}_{7-\delta} \delta$ Fulms Fabricated From Metallo-Organic Precursors", C. Venkatraman and G. L. Ledl, in preparation.

51. "Temperature Range for Critical Scaling Behavior in YBCO Thin Fulms", J. Deak, M. J. Darwin, and M. McElfresh, submitted.

52. "Evidence for Two Continuous Vortex Phase Transitions in $\mathrm{Tl}_{2} \mathrm{Ba}_{2} \mathrm{CaCu}_{2} \mathrm{O}_{7}$ Thin Films", J. Deak, M. McElfresh, D. W. Face, and W. L. Holstein, submitted to Phys. Rev. B Rapid Comm.

53. "Low-Temperature Electronic Properties of the $\mathrm{La}_{n+1} \mathrm{NinO}_{3 n+1}$ $(n=2,3$ and $\infty)$ System: Evidence for a Crossover from Fluctuating valence to Fermi Liquid-like Behavior", K. Sreedhar, M. McElfresh, D. Perry, D. Kim, P. Metcalf, and J. M. Honig, submitted.

54. "Single Electron Tunneling in THBa2(Ca0.8Y0.2) $\mathrm{Cu}_{2} \mathrm{O}_{7}$ Thin Films at 10K", T. G. Miller, R. Relfenberger, M. McElfresh, D. W. Face and W. L. Holstein, submitted. 


\section{MISCON THESES}

\section{January 1993 - December 1993}

\section{S. DEGREES}

1. Cynthia Smotzer, M. S. Thesis, Department of Physics, Indiana University, 1993. (Barter Group)

2. "Neutron Studies on High Tc Superconductors and Permanent Magnets", H. Xie, M. S. Thesis, University of Missouri-Columbia, August 1993. (Telon, Farmer Group)

3. Mark McClorey, M. S. Thesis, Department of Physics, University of Notre Dame, June 1993. (Tomasch, Furdyna Group)

4. "Crystal Growth and Characterization of the Nickel Sulfide, Heazlewooddite", Patricia A. Metcalf, M. S. Thesis, Department of Chemistry, Purdue University, August 1993. (Honig Group)

\section{DOCTORATE DEGREES}

1. Vincent DiStasi, Doctor of Philosophy, Department of Physics, Indiana University, December 1993. (Baxter Group)

2. "Studies of the Effects of Processing and Chemical Substitutions on the Microstructure and Properties of Melt Textured YBa2 $\mathrm{Cu}_{3} \mathrm{O}_{7}$ ", David Balkin, Doctor of Philosophy, Department of Engineering. University of Notre Dame, December 1993. (McGinn Group)

3. "Millimeter Microwave Properties of High-Temperature Superconductors", Eric K. Moser, Doctor of Philosophy, Department of Physics, University of Notre Dame. (Tomasch, Furdyna Group)

4. "Investigation of Transport Properties of Conventional and High Tc Superconducting Weak Link Devices", Steven T. Herbert, Doctor of Philosophy, Department of Physics, Ohio State University, August 1993. (Garland Group)

5. "Experimental Studies on Penetration Depth in YBa2 $\mathrm{Cu}_{3} \mathrm{O} 7-\delta$ Superconductors", Ju Young Lee, Doctor of Philosophy, Department of Physics, Ohio State University, December 1993. (Lemberger Group) 
APPENDIX I

RESEARCH PROGRAM LISTING

Area I:

New and Improved High $T_{c}$ Superconducting Materials: Phase Relations, Reactions, Synthesis and Processing

PI

Untv

Title

Funding

Affliations

Students \&

Status

D. V. Baxter

IU

Low Temperature Routes to

Processing of High-Temperature

105,000

Advanced Technology
Materials, Inc.

Professtonals

K. G. Caulton

Superconductors

Vincent DIStas1

Wen Chung Chiang

Cynthia Smotzer

Dr. Zlling Xue

Dr. Sylvain Dutremez

D. awarded 1993

Ph.D. anticlpated 1994

M.S. awarded 1993

K. M. Choudhary ${ }^{\circ}$ D

Study of Compositional Modulation

carry over

in High Temperature Superconductor

Chungling Zhong

Structures and Its Influence on their

P. Seshadr

Superconducting Properties
P. J. McGinn
ND
Microstructural Dependence of
Critical Current Density in Melt
Textured YBa2 $\mathrm{Cu}_{3} \mathrm{O}_{6+x}$
Superconductors

129,948

P. K. Gallagher* OSU Growth of Single Crystals and/or

Textured Specimens of High- $T_{C}$

Superconductors using Oxygen Seeding

K.H. Sandhage

OSU Processing of Textured Super-

conducting Oxdde/Noble

90,000 Metal Microlaminates

Jewen Bae

Ph.D. anticipated 1995 Ph.D. anticipated 1994 Left MSE Program 8/93

P.J. McGinn

Anisotropic Grain Growth and

Isothermal Grain Alignment of Bulk.

Superconducting Oxides

100,390

K. H. Sandhage

\section{David Balkdn}

Survankar Sengupta

Varanast Chakrtpan!

Hal-feng Yang

J. Kirksey

Chan Joong KIm

Styoung Chol

Thawl Iwagosh!

S. Vlayannur

Marc Black

$$
\text { General Cor }
$$

Rıbbon Technolog

Corporation
Ph.D. awarded 12-93

Ph.D. anticlpated 5-94

Ph.D. anticipated 8-94

Ph.D. anticipated 5-97

Technictan

Postdoctoral Assoctate

Ph.D. antictpated 6-94

Ph.D. anticipated 1994

Ph.D. anticipated 1-96

Ph.D. anticipated 5-95

\section{Yong-Y1 Sh1}

David Chmielewsk

Holly Miron
Left Untv. 7-93

M.S. antictpated 6-94

M.S. antictpated 6-95 


\section{J. M. Honig \\ J. Spalek \\ PU \\ Pair Breaking Phenomena in the

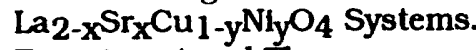 \\ Experiment and Theory}

G.L. Liedl PU

A.L. Bement, Jr.

Processing and Texture

83,366

Development in $\mathrm{YBa}_{2} \mathrm{Cu}_{3} \mathrm{O}_{7-\delta}$

Films Synthesized from Metallo-Organic Precursors

K.P. Trumble M.W. McElfresh

K.J. Bowman

PU

High Temperature Superconducting Tubes for Magnetic Shielding Applications
116,500
H. Sato*
Study of Naturally Modulated High
carry over

$T_{c}$ Superconducting Compounds

Area II:

Figh Tc Superconductor Tranoport Properties: Controlling/Limiting Factor S. M. Girvin

J. R. Clem*

V. G. Kogan

Meera

Chandrasekhar
A. H. MacDonald IU
Quantum and Statistical

Mechanics of High $T_{c}$ Superconductors and Flux Line Liquids
Quantum Magnetics/ Guantum Design
P. Metcalf

K. Sreedhar

P. Metclaf

C. Venkatraman

S. Mansour

D. Perry

Shi Li

Greg Stelnlage

N. Medendorp

Darin Aldrich

Chad Peterson

Amy Trissler

Timothy Brent

H. Zhang

J.G. Chen
High-Temperature Superconductors

71.400

AT\&T Bell Lab

Electrical and Optical

Investigations of Pristine and

Doped $\mathrm{YBa}_{2} \mathrm{Cu}_{4} \mathrm{O}_{8}$
M.S. awarded 8-93

Postdoctoral Associate Professional

Ph.D. anticipated 1994

Professional

Professional

Ph.D. anticipated 1996

M.S.E. anticlpated 5-94

M.S.E. anticipated 1995

B.S.E. awarded 5-93

B.S.E. awarded 5-93

B.S.E awanded 5-93

B.S.E. anticlpated 5-94

Ph.D. anticipated 1994 Postdoctoral Researcher
Jun Hu

Yong Wang

Ross Hyman

Hiroshi Akera

Mats Wallin

Maamar Benkraouda Andrew Y. Simonov

Dale Payne
Ph.D. anticipated 1994 Ph.D. anticipated 1995 Ph.D. anticipated 1995 Visiting Scientist Visiting Scientist

Ph.D. anticipated 1994 Postdoctoral Fellow

"Project Withdrawn 
W. B. Yelon

J. W. Farmer

UM- Neutron Diffraction and Defect

Studies of High $T_{C}$ Superconductor

70,000

H. A. Blackstead ND

Microwave and Optical Studies of

High-Temperature Superconductors:

Flux-Lattice Dynamics, Crystal Anisotropy,

Flux Pinning and Photo-Modulated Superconductivity
A. E. Muller**
ND
Superconduct
69,986

S. Bandyopadhyay

S. T. Ruggiero*

Transport Properties of YBCO Thin Films

carry over

Magneto-Dissipation in High-

Temperature Superconductors

o J. K. Furdyna

OSU High $T_{c}$ Superconducting

Composites, Arrays and Crystals

J. C. Garland

G. P. Lafyatis

OSU Atomic Beam Studies of Vortex

Lattices at Superconductor Surfaces

T. R. Lemberger

OSU Device-related Properties of Doped

$\mathrm{YBa}_{2}\left(\mathrm{Cu}_{1-\mathrm{x}} \mathrm{M}_{\mathrm{X}}\right)_{3} \mathrm{O}_{7-\delta}$ Films
49,000

118,605

H. XIe

Ann Klunzingeer

Pleder Beell

D.B. Pulling

Duofeng Yue

Gautam Banerjee

Chunling Zhong

E.K. Moser

Mark McClorey

S.T. Herbert

Y.M. Wan

John Markus

Matt Trawick

Sergio Ammirata

C. Ash

S.E. Hebboul

D.C. Harris

Hu Jong Lee

73,500

62,000
S. Dapore-Schwartz Harold Haughlin

Ju Young Lee

Eric Ulm

Jack Kessler
M.S. awarded 8-93

Graduate Student

Graduate Student

Professional Specialist

Ph.D. anticipated 1994 Postdoctoral Assoctate

Graduate Student

Ph.D. awarded 8-93

M.S. awarded 6-93

Ph.D. awarded 8/93

Ph.D. anticipated 1994

Ph.D. anticlpated 1996

Ph.D. anticlpated 1996

Ph.D. anticlpated 1997

M.S. anticipated 1994

Professional

Postdoctoral Assoclate

Vlsitor

Ph.D. anticipated 1994

Ph.D. anticipated 1996

Ph.D. awarded 1993

Graduate Research Asst.

Graduate Research Asst. 


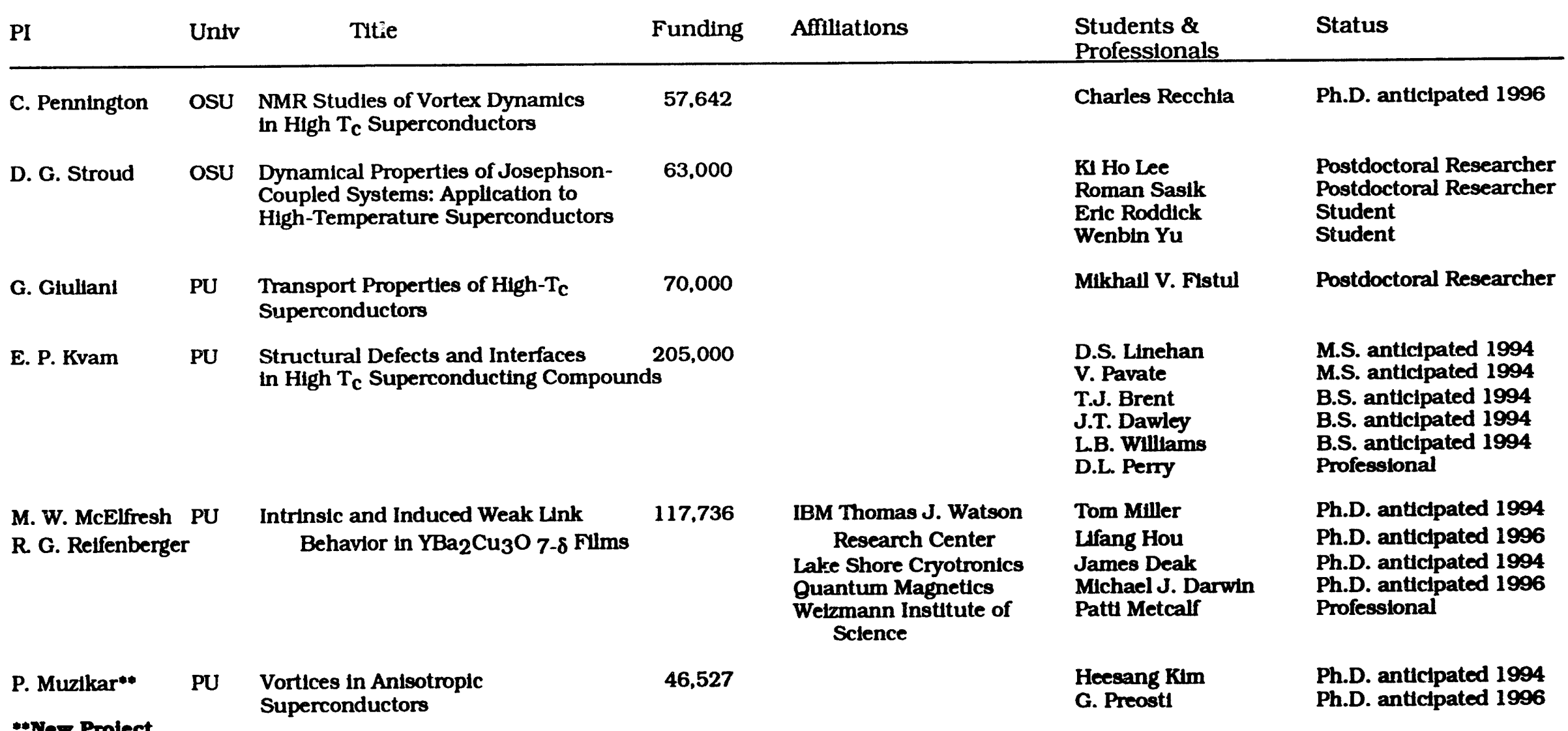




\section{APPENDIX II \\ Cooperative Laboratory Interactions \\ January 1993 - December 1993}

COMPANY/CONTACT

\section{SEMINAR AND GUEST SPEAKERS}

James D. Jorgensen

Argonne National Laboratory

Dean E. Peterson

Los Alamos National Laboratory

$\infty$

Dr. Paul Muzlkar

Purdue University

Dr. David Gaskell

Purdue University

Dr. C. J. van der Beek

Argonne National Laboratory

Dr. David G. Stroud

Ohio State University

Dr. Tom Lemberger

Ohio State University

M. V. Indenbom

Institute of Solid State Physics

Russian Academy of Science, Moscow, Russia

ACTIVITY

MISCON PI/LOCATION

Guest Speaker at Group Meeting

Guest Speaker at Group Meeting

Invited Seminar Speaker

Invited Seminar Speaker

Invited Seminar Speaker

Invited Seminar Speaker

Invited Seminar Speaker

Invited Seminar Speaker

Invited Seminar Speaker
Columbla, MO

Columbia, MO

Vladimir Kogan

Iowa State Untversity

Purdue University

Purdue University

Purdue Untversity

Purdue University

Indiana University

Purdue University 
Martin Nikolo

St. Louls University

St. Louis, MO

Allen Goldman

University of Minnesota

Valerti Vinokur

Argonne National Laboratory

Masakd Suenaga

Brookhaven National Laboratory

Marko Ledvij

Iowa State University

Alex Gurevich

University of Wisconsin-Madison

Madison, Wisconsin

Alvaro Sanchez

Universitat Autonoma Barcelona Spain

Hugo Safar

AT\&T Bell Labs

Borls Amonzon

Russtan Scientific Center

Moscow, Russia

Dr. A. Gurevich

Untversity of Wisconsin-Madison

Madison, Wisconsin

Dr. Milind N. Kunchur

Oak Ridge National Laboratory

Oak Ridge. Tennessee
Invited Seminar Speaker

Purdue University

Guest Speaker at Group Meeting and Workshop Speaker

Workshop Speaker

Workshop Speaker

Workshop Speaker

Workshop Speaker

Workshop Speaker

Workshop Speaker

Invited Seminar Speaker

Invited Seminar Speaker

Invited Seminar Speaker
University of Notre Dame

Untversity of Notre Dame

Untversity of Notre Dame

Untversity of Notre Dame

Untversity of Notre Dame

Untversity of Notre Dame

Untversity of Notre Dame

Purdue Untversity

Purdue University

Purdue Untversity 


\section{PROGRAMMATIC EXCHANGE PROGRAM}

Jewan Bae

Choudhary Group

Untversity of Notre Dame

Dimitri Xenikos

Lemberger Group

Ohio State University

Mike Darwin, Jim Deak, and Shi LI

McElfresh Group

Purdue University

Parthasarathy Seshadri

Choudhary Group

Untversity of Notre Dame

Chakrapani Varanas!

Untversity of Notre Dame

\section{CO-OP EFFORTS/COLABORATIONS}

Daniel Arovas

UCSD

San Diego, CA

John Berlinsky

McMaster

Min-Chul Cha

University of Kentucky

David Stroud

Ohio State University

Columbus, $\mathrm{OH}$
Measurements of Transport Properties

M. McElfresh Lab

Purdue University

Use of Laser Ablation System

Discussions on Setting up and Maintaining

Electromagnetically Shielded Room

Study of the crystal structure of $\mathrm{Er}_{5} \mathrm{Ba}_{7} \mathrm{Cu}_{12} \mathrm{O}$

thin fllms on various substrates

Perform TEM Work

Discusstons on Microscopic and

Macroscopic Properties

Discussions on Microscopic and

Macroscopic Properties

Field-tuned Superconductor-insulator Transition

Vortex Glass Simulations
M. McElfresh Lab Purdue University

S. Ruggiero Lab Untversity of Notre Dame

G. L. Lledl Lab Purdue University

\section{Purdue University \\ E. Kvam Lab}

A. H. MacDonald S. M. Girvin Indiana University

A. H. MacDonald

S. M. Girvin

Indiana University

A. H. MacDonald

S. M. Girvin

Indiana University

A. H. MacDonald

S. M. Girvin

Indiana University 


\section{A. Peter Young}

UCSC

Matthew P. A. Fisher

UCSB

Michael Norman

Argonne National Lab

Argonne, IL

M. J. Kramer

Iowa State University

\section{A. Kirk}

Argonne National Laboratory

P. Allenspach

Untversity of Callfornia

San Diego, CA

J. J. Neumeier

Los Alamos National Laboratory

J. Schilling

Washington University

St. Louis, MO

J. Diederichs

Washington University

St. Louis, MO

\section{W. J. James}

University of Missourt-Rolla

J. J. Rhyne

University of Missouri-Columbia
Vortex Glass Transition in 2D

Collaborative Research

Microscopic Study of Superconductors in High Fields

\section{Collaboration}

Collaboration

Collaboration

Collaboration

Collaboration

Collaboration

Collaboration

Collaboration
A. H. MacDonald

S. M. Girvin

Indiana University

A. H. MacDonald

S. M. Girvin

Indiana University

A. H. MacDonald

S. M. Girvin

Indiana University

W. B. Yelon

J. W. Farmer

University of Missouri-Col

J. W. Farmer

Untversity of Missouri-Col

W. B. Yelon

University of Missouri-Col .

W. B. Yelon

University of Missouri-Col

W. B. Yelon

University of Missourt-Col

w. B. Yelon

University of Missouri-Col

W. B. Yelon

Untversity of Missouri-Col

W. B. Yelon

University of Missouri-Col 
J.Z.Wu

University of Kansas

Y. Xin

Midwest Superconductivity, Inc.

Lawrence, KS

\section{K. W. Wong}

University of Kansas and

Midwest Superconductivity, Inc.

Lawrence, KS

P. Dal

Oak Ridge National Laboratory

R. W. McCallum

Iowa State University

J. M. Honig

Purdue University

J. D. Dow

Artzona State

Dr. Donglu Sh1

Argonne National Lab

Rich Fletcher

Wright Patterson AFB

Parthasarathy Seshadri

Untversity of Notre Dame

D. Rudman

NIST

Boulder, $\mathrm{CO}$
Collaboration

Collaboration

Collaboration

Collaboration

Collaboration

Collaboration

Collaboration

Joint Project

Collaboration on Transport and

Microwave Properties

YBCO Superconductor Multilayers

Thin Film YBCO
W. B. Yelon

University of Missouri-Col

W. B. Yelon

University of Missourt-Col

W. B. Yelon

University of Missour-Col

W. B. Yelon

University of Missourt-Col

w. B. Yelon

University of Missouri-Col

W. B. Yelon

Untversity of Missourt-Col

H. Blackstead

Untversity of Notre Dame

P. J. McGinn

Untversity of Notre Dame

S. T. Ruggiero

Untversity of Notre Dame

S. T. Ruggiero

Untversity of Notre Dame

S. T. Ruggiero

Untversity of Notre Dame 
Beth Baumert

Westinghouse

Pittsburgh, PA

Peter Rosenfeld

Advanced Fuel Research

W. Hartford, Conn.

\section{Greg Hey-Shipton}

Lincoln Bourne

Superconductor Technologies, Inc.

Mike McElfresh

Purdue University

Steve Schwarzbek

Claire Petiette-Hall

TRW Space and Technology

Redondo Beach, CA

Mark Coffey

NIST

Boulder, CO

John R Clem

Ames Lab

Iowa State University

M. J. Kramer

Iowa State University

Gordon Roesler

NRL

J. Garland

Ohio State University

Rutherford Backscattering Facillty Cornell University
Collaboration on YBCO Step-edge Studies

Exchange of FAR/Josephson Junction Results

YBCO Film Preparation and also

Contributions to edited work "Superconductor

Devices Handbook"

Loan of Equipment

for Low-temperature Experiments

YBCO Thin Film Collaborations

YBCO Thin Film Collaborations

Comparisons of Microwave Data

Collaborations

Collaborations

Collaborations

Improvement of the Stoichiometry

of Codeposited Films
S. T. Ruggiero

Untversity of Notre Dame

S. T. Rugglero

Untversity of Notre Dame

S. T. Ruggiero

Untversity of Notre Dame

S. T. Ruggiero

Untversity of Notre Dame

W. J. Tomasch

Untversity of Notre Dame

w. J. Tomasch

Untversity of Notre Dame

W. J. Tomasch

Untversity of Notre Dame

P. Gallagher

Ohio State Untverstty

G. Lafyatis

Ohio State Untversity

G. Lafyatis

Ohio State Untversity

T. R. Lemberger

Ohio State University 
A. H. MacDonald

S. M. Girvin

Indiana University

D. Stroud

Ohio State University

Dr. Bart Riley

American Superconductor Corp.

Brian Maple

Untversity of California

San Diego, CA

Dr. KI II. Lee

Syracuse University

M. McElfresh Group

Purdue Untversity

M. McElfresh Group

Purdue University

C. Broholm

Johns Hopkins University

T. Rosenbaum

Untversity of Chicago

J. Brill

Untversity of Kentucky

P. Eklund

University of Kentucky

J. Aeppli

AT\&T Bell Laboratories
Discussions on vortices

Interpretations of Data

Collaborative Experiments

Collaboration on Transport and Magnetic Properties

\section{Collaboration}

Weak Link Properties of Graln Boundaries in $\mathrm{BSCCO}$ and $\mathrm{YBCO}$

Critical Transport of Junctions Artificially Manufactures out of Narrow Channels

Collaborative Research

Collaborattve Research

Collaborattive Research

Collaborative Research

Collaborative Research
T. R. Lemberger

Ohio State Untversity

T. R. Lemberger

Ohio State Untversity

K. Sandhage

Ohio State Untversity

D. Stroud

Ohio State University

D. Stroud

Ohto State Untversity

G. Gluliani

Purdue Untversity

G. Glultani Purdue Untversity

J. Honig Purdue Untversity

J. Honig Purdue Untversity

J. Honig Purdue Untversity

J. Honig Purdue Untversity

J. Honig

Purdue Untversity 


\section{J. Acrivos}

San Jose State University

\section{Stassis}

lowa State Untversity

J. W. Farmer

University of Missourt-Columbia

\section{w. Yelon}

University of Missouri-Columbla

Dr. U. Balachandran

Argonne National Lab

Tom Lemberger

Ohio State Untversity

Dr. Jim Eckstein

Varlan Research Laboratories

Palo Alto, CA

Dean Peterson

Los Alamos National Lab

Los Alamos, NM

Jon Martens

Conductus

Alan Kleinsasser

IBM T.J. Watson Research Center

Yorktown Heights, NY

John Neumeier

Los Alamos National Lab

Los Alamos, NM
Collaborattve Research

Collaborative Research

Collaborative Research

Collaborattve Research

Collaboration on joint project

Discussions on penetration depth calculations

Collaborations

Collaborations

Collaborations

Collaborations

Collaborations
J. Honig

Purdue Untversity

J. Honig

Purdue Untversity

J. Honig

Purdue Untverstty

J. Honig

Purdue Untversity

E. Kvam

Purdue Untversity

P. Muzikar

Purdue Untversity

R. Retfenberger

M. McElfresh

Purdue Untverstty

K. Trumble

Purdue Untverstty

K. Trumble

Purdue Untverstty

M. McElfresh

Purdue Untversity

M. McElfresh

Purdue Untverstty 
Ross Muenchausen

Los Alamos National Lab

Los Alamos. NM

Dean Face

DuPont Central Research

Wilmington, DC

John R. Clem

Iowa State University

Ames, IA

Mike McHenry

Carnegie-Mellon University

Pittsburgh, PA

\section{SAMPLE EXCEANGE/COLIABORATIONS}

P. Boolchand

University of Cincinnati

B. Dabrowskd

Argonne National Lab

J. Schilling

Washington University

T. Venkatesan

University of Maryland

S. Bandyopadhyay

Untversity of Notre Dame

Dr. Xlao Ping Jlang

Morris Research, Inc.

Berkeley. CA

\section{Collaborations}

Collaborations

Collaborations

Collaborations

Sample Exchange

Sample Exchange

Expertment on Pressure Dependencles of Raman modes in TI 2201

Discussion of collaboration

Collaboration

Sample Exchange/Joint Project
M. McElfresh

Purdue Untversity

M. McElfresh

Purdue Untversity

M. McElfresh

Purdue Untversity

K. Trumble

Purdue Untversity

M. Chandrasekhar Untversity of Missourt-Col

M. Chandrasekhar Untversity of Missouri-Col

M. Chandrasekhar Untversity of Missourt-Col

M. Chandrasekhar Untversity of Missourt-Col

M. Chandrasekhar Untverstty of Missourt-Col

P. J. McGinn

Untverstty of Notre Dame 
Prof. Robert Snyder

Alfred Untversity

Dr. Jeffrey A. Eastman

Argonne National Laboratory

Dr. Richard Ricker

NIST

Gaithersburg, Maryland

Dr. Meera Chandrasekhar
NIMET Industries, Inc.

Sample Exchange/Joint Project

Charactertzing Structures using SEM, TEM

Anodization and Fabrication of Templates

Technology Transfer and Adaptation of the Anodization Process

Possible Collaboration on Examination of Structures with Raman Spectroscopy

$\mathrm{Bi}_{2} \mathrm{Sr}_{2} \mathrm{CaCu}_{2} \mathrm{O}_{8-\mathrm{y}}$ Samples

$\mathrm{Bi}_{2} \mathrm{Sr}_{2} \mathrm{CaCu}_{2} \mathrm{O}_{8-\mathrm{y}}$ Samples

Ag/YBa2 $\mathrm{Cu}_{3} \mathrm{O}_{7}$ Arrays

Thomas R. Lemberger Ohlo State Untversity

Dale Van Harlingen

Untversity of Illinois

Urbana-Champaign

Charles Pennington

Ohio State University

Steve Foltyn and Xindi Wu Los Alamos National Lab
$\mathrm{Bi}_{2} \mathrm{Sr}_{2} \mathrm{CaCu}_{2} \mathrm{O}_{8-\mathrm{y}}$ Samples

Single Crystals of $\mathrm{YBa}_{2} \mathrm{Cu}_{3} \mathrm{O}_{7-\delta}$

Laser Ablated Fllms of YBCO
P. J. McGinn Untversity of Notre Dame

A.E. Miller. B. Das, \& S. Bandyopadhyay Untverstty of Notre Dame

A.E. Muller, B. Das,

\& S. Bandyopadhyay

Untversity of Notre Dame

A.E. Miller, B. Das,

\& S. Bandyopadhyay

Untverstty of Notre Dame

A.E. Miller. B. Das \& S. Bandyopadhyay Untverstty of Notre Dame

J. C. Garland Ohto State Untverstty

J. C. Garland Ohio State Untversity

J. C. Garland Ohio State Untverstty

J. C. Garland Ohio State Untversity

G. Lafyatis Ohio State Untversity

T. R. Lemberger Ohio State Untversity 
Mike McElfresh

Purdue University

Julla Phililps

AT\&T Bell Labs

P. Gallagher

Ohio State University

Brian Maple

University of California-San Diego

San Diego, CA

James Garland

Ohio State University

Alan Hermann

Untversity of Colorado

\section{INDUSTRIAL CONTACTS}

\section{AT\&T Bell Labs}

Dr. D. Bishop

Dr. K. W. Wong

Midwest Superconductors, Inc.

Lawrence, KS

Dean Face

DuPont

Dr. David Rudman

High Temperature Superconductors

NIST

Boulder, $\mathrm{CO}$
Laser Ablated Films of YBCO

Drawings for Substrate Heater

Films of pure YBCO for comparison

Use of Lab for Fabrication of Powder Precursors

Puswder and Single Crystal samples of

${ }^{17} \mathrm{O}$-enriched $\mathrm{YBa}_{2} \mathrm{Cu}_{3} \mathrm{O}_{7-\delta}$

Single Crystals of BSSCO (2212)

Single Crystais of THBaCuO (2202)

Discusstons on Expertmental

Results

Industrial Affiliate Discussions

Industrial Affiliate Discussions

Industrial Affiliate Discussions
T. R. Lemberger

Ohio State University

T. R. Lemberger

Ohio State Untversity

T. R. Lemberger

Ohio State Untversity

C. Pennington

Ohto State Untverstty

C. Pennington

Ohio State Untverstty

C. Pennington

Ohio State Untversity

\section{G. Kogan}

Iowa State Untversity

w. B. Yelon

J. W. Farmer

Untversity of Missourt-Col

S. T. Ruggiero

Untversity of Notre Dame

S. T. Rugglero

Untversity of Notre Dame 
Lincoln Bourne

Greg Hey-Shipton

Superconductor Technologies

Santa Barbara, CA

Beth Baumert

Westinghouse Science and Technology Center Pittsburgh, PA

National Nanofabrication Facility

Cornell University

Ithaca, NY

Mike Tomsic

Plastronic, Inc.

Troy, $\mathrm{OH}$

Dr. C. E. Oberly

Wright Patterson Air Force Base

\section{INDUSTRLAL IFFILIATES}

Advanced Technology Materials, Inc. John Steinbeck

Danbury, CT

\section{AT\&T Bell Labs}

Dr. David J. Bishop

Murray Hill, NJ

Bellcore

Dr. R Ramesh

Red Bank, NJ

IBM Thomas J. Watson Research Center

Dr. Stuart Bermon

Yorktown Heights, NY
Industrial Afflliate Discussions

Industrial Affiliate Discussions

Sample Fabrication

Industrial Afflilate Discussions

Informal Collaboration with

goal of Formal Affiliate Agreement

Written Agreement

Written Agreement

Written Agreement

Written Agreement
S. T. Ruggiero

Untversity of Notre Dame

S. T. Ruggiero

Untversity of Notre Dame

J. C. Garland

Ohio State University

K. Sandhage

Ohio State University

E. Kvam

Purdue University

K. G. Caulton

Indiana University

J.R Clem, V.G. Kogan

Iowa State .University

E. Kvam, M. McElfresh

Purdue Untversity

M. W. McElfresh Purdue University 
Intermagnetics General Corporation

Dr. Pradeep Haldar

Gutlderland. NY

Lake Shore Cryotronics, Inc.

B. C. Dodrill

Westerville, $\mathrm{OH}$

National Institute of Standards and Technology Written Agreement Dr. Robert A. Kamper and

Dr. M. W. Coffey

Quantum Magnetics

Dr. Ronald E. Sager and Dr. Barry Lindgren

San Dlego, CA

\section{Ribbon Technology Corporation}

Dr. Tom Gaspar

TRW Superconducttvity Research

Stephen M. Schwarzbek

Redondo Beach, CA

\section{Dr. ElHa Zeldov}

The Weizmann Institute of Science

Rehovot. Israel

\section{FOREIGN INTERACTIONS}

Hiroshı Akera

Hokkaldo University

Japan

Mats Wallin

KTH

Stockholm. Sweden
Equipment Access

Written Agreement

Written Agreement

Equipment Access

Written Agreement

Written Agreement

Studying Pair Tendencies in the Extreme Strong Magnetic Fleld Limit and Visit

Joint Project and Visit
K. Sandhage, P. McGinn

Ohio State University

Untversity of Notre Dame

MISCON Researchers

W. J. Tomasch

Untversity of Notre Dame

M. W. McElfresh

Purdue Untversity

K. Sandhage, P. McGinn

Ohio State University

Untversity of Notre Dame

W. J. Tomasch

Untversity of Notre Dame

M. W. McElfresh

Purdue Untversity

A. H. MacDonald

S. M. Girvin

Indiana University

A. H. MacDonald

S. M. Girvin

Indiana University 


\section{A. E. Schlogl}

University of Münich

Münich, Germany

\section{Allgeter \\ University of Münich \\ Münich, Germany}

R. Sieburger

University of Mūnich

Münich, Germany

S. K. Malik

Tata Inst. for Fundamental Research

Bombay, India

\section{H. Jahns}

Tata Inst. for Fundamental Research

Bombay, India

R. Prasad

Bhabha Atomic Research Center

K. Adhikary

Bhabha Atomic Research Center

N. C. Soni

Bhabha Atomic Research Center

VIP, Inc

India

IIT

Bombay, India

G. Lander

Euratom, Karlsruhe

\section{Collaboration}

Collaboration

Collaboration

Collaboration

Collaboration

Collaboration

Collaboration

Collaboration

Anodized Samples

Anodized Samples

Collaborative Research
W. B. Yelon

University of Missouri-Col

W. B. Yelon

University of Missouri-Col

\section{w. B. Yelon}

University of Missouri-Col

W. B. Yelon

University of Missourd-Col

W. B. Yelon

Untversity of Missouri-Col

\section{W. B. Yelon}

University of Missouri-Col

W. B. Yelon

University of Missouri-Col

w. B. Yelon

Untversity of Missouri-Col

A. E. Miller

Untversity of Notre Dame

A. E. Miller

Untversity of Notre Dame

J. Honig

Purdue University 
Dr. Marcin Konczykowskd

Ecole Polytechnique

Palaiseau, France

\section{Ell Zeldov}

The Welzmann Institute of Science

Rehovot, Israel

\section{Yosi Yeshurun}

Bar-Ilan University

Ramat-Ganz, Israel
Joint Projects and Visits

Collaboration

Collaboration
M. W. McElfresh Purdue University

M. W. McElfresh

Purdue Untversity

M. W. McElfresh

Purdue University 


\section{APPENDIX III}

ORGANIZATION

January 1, 1992 - December 31, 1993

\section{A. SPONSOR}

Robert Gottschall

Program Manager
Division of Materials Sciences

Office of Basic Energy Sciences

U.S. Department of Energy

$301-353-3428$

\section{B. OFFICE OF THE DIRECTOR}

Arden L. Bement, Jr. Purdue University

$317-494-5567$

Director

Michael McElfresh

Purdue University

317-494-5567

Assistant Director

Purdue University

317-494-5567

Rebecca Herr

Assistant to the Director

C. COMMnTeEs

Steering Committee:

Robert Greenkorn

Purdue University

$317-494-6840$

Chair

Vice President for Research

Joseph A. Franklin Indiana University

812-855-6394

Director

Government Policy and

Federal Relations

Stuart Hadley

Iowa State University

515-294-0523

Assistant Director

Office of Science Policy

and Research

Nathan O. Hatch

Vice President for

University of Notre Dame

219-239-6291 
Richard S. Stoddard The Ohio State University

614-292-1582

Vice President for Research

Ronald J. Turner

Special Assistant

to the President

Newton Cattell

Ex Officio Member

Gerald L. Liedl

Ex Officlo Member

Arden L. Bement, Jr.

Director

Ex Officlo Member

\section{Program Committee:}

Gerald L. Liedl

Chair

Arden L. Bement, Jr. Purdue University

Director

Michael McElfresh

Assistant Director

Rebecca Herr

Assistant to the Director

(non-voting member)

Paul McGinn

Leader, Group I

John R. Clem

Leader, Group $\mathbf{I}$

Meera Chandrasekhar

James C. Garland

Jurgen M. Honig

David V. Baxter

John Mundy

Program Manager

Purdue University

Purdue University

Purdue University

Purdue University

Purdue University
University of Missouri-Columbia

Midwestern Universities Alliance

317-494-5567

317-494-5567

$317-494-5567$

317-494-5567

University of Notre Dame

$219-239-6151$

Iowa State University

515-294-4223

University of Missouri - Columbia

314-882-2619

The Ohio State University

614-292-7277

Purdue University

$317-494-5279$

Indiana University

812-855-8337

Division of Materials Sciences

Office of Basic Energy Sciences

Department of Energy 


\section{PRINCIPAL INVESTIGATORS}

David V. Baxter

Kenneth G. Caulton

Malcolm H. Chisholm

Allan H. MacDonald

Steven M. Girvin

John R. Clem

Vladimir G. Kogan

Meera Chandrasekhar

W.B. Yelon

J.W. Farmer

Howard A. Blackstead

K.M. Choudhary

S.T. Ruggiero

Paul J. McGinn

Albert E. Miller

Biswajit Das

Supriyo Bandyopadhyay

Walter J. Tomasch

Jacek F. Furdyna

Patrick K. Gallagher

James

C. Garland

Gregory P. Lafyatis

Thomas $\mathbf{R}$ Lemberger

Charles Pennington

Ken $H$. Sandhage
Indiana University

Indiana University

Indiana University

Indiana University

Indiana University

Iowa State University

Iowa State University

University of Missouri - Columbia

University of Missouri - Columbia

University of Missouri - Columbia

University of Notre Dame

University of Notre Dame

University of Notre Dame

University of Notre Dame

University of Notre Dame

University of Notre Dame

University of Notre Dame

University of Notre Dame

University of Notre Dame

Ohio State University

Ohio State University

Ohio State University

Ohio State University

Ohio State University

Ohio State University
812/855-8337

$812 / 855-4798$

812/855-6606

812/855-1304

812/855-3735

$515 / 294-4223$

$515 / 294-8080$

$314 / 882-2619$

$314 / 882-5236$

$314 / 882-5249$

219/631-7078

219/631-6277

219/631-7463

219/631-6151

219/631-8307

219/631-7379

219/631-4393

219/631-6152

219/631-6741

$614 / 292-3010$

$614 / 292-7277$

$614 / 292-2286$

614/292-7799

$614 / 292-6456$

$614 / 292-2491$ 
David G. Stroud

Gabriele F. Giuliani

J.M. Honig

J. Spalek

Eric P. Kvam

G.L. Liedl

Arden L. Bement, Jr.

Paul F. Muzikar

R. Reifenberger

M. McElfresh

H. Sato

Kevin P. Trumble

Keith J. Bowman
Ohio State University

Purdue University

Purdue University

Purdue University

Purdue University

Purdue University

Purdue University

Purdue University

Purdue University

Purdue University

Purdue University

Purdue University

Purdue University
$614 / 292-8140$

317/494-5384

317/494-5279

317/494-0218

317/494-4097

317/494-5567

317/494-5567

317/494-0706

317/494-3032

3) 7/494-6605

317/494-4099

317/494-4114

317/494-6316 

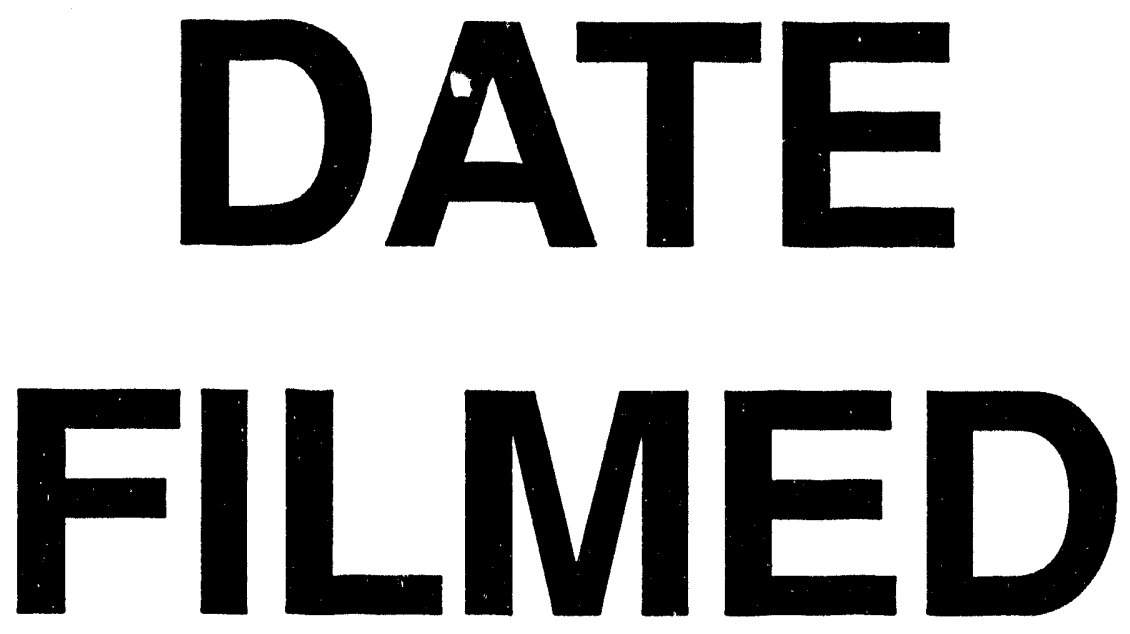

$6 / 28 / 94$
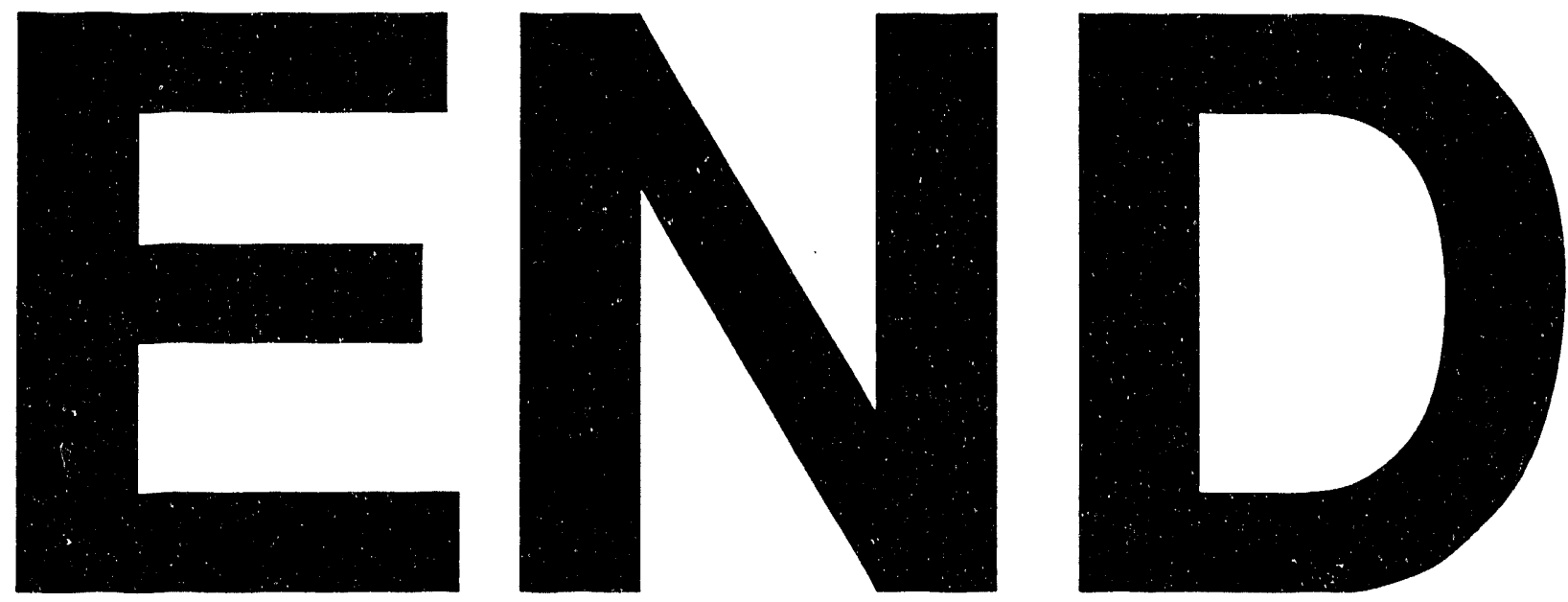
